\title{
Thymic macrophages consist of two populations with distinct
}

4 Tyng-An Zhou ${ }^{1}$, Hsuan-Po Hsu ${ }^{1}$, Yueh-Hua Tu²,3, Chih-Yu Lin ${ }^{1}$, Nien-Jung

5 Chen $^{1}$, Jin-Wu Tsai ${ }^{4}$, Ellen A. Robey ${ }^{5}$, Hsuan-Cheng Huang ${ }^{2,6}$, Chia-Lin Hsu ${ }^{1}$,

6 and Ivan L. Dzhagalov ${ }^{*}$

$7 \quad{ }^{1}$ Institute of Microbiology and Immunology, National Yang-Ming University, Taipei, Taiwan

8 2Bioinformatics Program, Taiwan International Graduate Program, Institute of Information

9 Science, Academia Sinica, Taipei, Taiwan

$10{ }^{3}$ Graduate Institute of Biomedical Electronics and Bioinformatics, National Taiwan University,

11 Taipei, Taiwan

$12 \quad{ }^{4}$ Brain Research Center, National Yang-Ming University, Taipei, Taiwan

13 DDivision of Immunology and Pathogenesis, Department of Molecular and Cell Biology,

14 University of California, Berkeley, CA, United States

$15{ }^{6}$ Institute of Biomedical Informatics, National Yang-Ming University, Taipei, Taiwan

17 *Correspondence to Ivan L. Dzhagalov: ivan.dzhagalov@nycu.edu.tw, ORCID: 0000-0001-

$18 \quad 9209-4582$

19 Mailing Address: Ivan L. Dzhagalov, Institute of Microbiology and Immunology, National

20 Yang-Ming Chiao-Tung University, \#155, Sec. 2, LiNong Street, Beitou, Taipei City, 11221,

21 TAIWAN 


\section{Abstract}

Tissue-resident macrophages are essential for protection from pathogen invasion and maintenance of organ homeostasis. The ability of thymic macrophages to engulf apoptotic thymocytes is well appreciated, but little is known about their ontogeny, maintenance, and diversity. Here, we characterized the surface phenotype and transcriptional profile of these cells and found out that they express typical tissue-resident macrophage genes yet

31 also exhibit organ-specific features. Thymic macrophages were most closely related to spleen red pulp macrophages and Kupffer cells and shared the expression of the transcription factor SpiC with these cells. Using shield chimeras, transplantation of embryonic thymuses, and fate mapping, we

35 found that three distinct waves of precursors generate thymic macrophages. Moreover, some of them proliferated in situ. Single-cell RNA sequencing

37 showed that the macrophages in the adult thymus are composed of two populations with distinct localization and origin. Altogether, our work defines the phenotype, origin, and diversity of thymic macrophages. 


\section{Introduction}

Tissue-resident macrophages are present in every organ and maintain local homeostasis through diverse functions ranging from protection against pathogens to tissue repair[1]. To perform their roles efficiently, macrophages acquire specialized phenotypes depending on the tissue microenvironment, and as a consequence, multiple subtypes exist, frequently within the same organ. For example, the spleen harbors red pulp macrophages specialized in red blood cell phagocytosis, marginal zone macrophages and metallophilic macrophages that are the first defense against blood-borne pathogens, $\mathrm{T}$ cell zone macrophages that silently dispose of apoptotic immune cells, and tingible-body macrophages that engulf less fit B cells in the germinal center[2system that allows enormous plasticity.

The last decade has seen a paradigm shift in our understanding of the development of tissue-resident macrophages. Contrary to the long-held belief that all macrophages derive from circulating monocytes[5], multiple studies have shown that many of them are long-lived cells with an embryonic origin that can maintain themselves in the tissues (reviewed in[6]). Three waves of distinct progenitors settle the tissues and contribute in various degrees to the resident macrophages in each organ. The first wave consists of yolk sac (YS)-

61 derived primitive macrophages that enter all tissues and establish the earliest macrophage populations $[7,8]$. In all organs, except for the brain and, partially,

63 the epidermis, primitive macrophages are replaced by the next wave consisting of fetal monocytes[9-12]. The third wave comes from hematopoietic stem cell (HSC)-derived monocytes that contribute to various degrees to the 
macrophage pool in different tissues. For example, these cells contribute little to the microglia in the brain, Langerhans cells in the epidermis, and alveolar macrophages in the lungs but substantially to most other organs[13-16]. Moreover, the kinetics and timing of HSC-derived monocytes infiltration vary in different parts of the body. For some macrophage populations, such as the arterial macrophages and subcapsular lymph node macrophages, monocytes replace embryonic macrophages soon after birth and self-maintain after that with little contribution from circulating cells[17,18]. Others, such as heart macrophages, osteoclasts, and pancreatic isles macrophages are progressively replaced at a low rate[14,19-23]. A third group, such as the macrophages in the dermis and most of the gut macrophages, are constantly replaced by blood monocytes with relatively fast kinetics[24,25]. These conclusions have been extended to many different macrophage populations such as Kupffer cells, liver capsular macrophages, red pulp macrophages, testicular macrophages, large and small peritoneal macrophages, and T zone macrophages in the lymph nodes[2,13,14,16,26-30].

The recent revitalization in macrophage research has yet to reach thymic macrophages. Although their prodigious phagocytic ability is well appreciated[31], little is known about the origin, diversity, and maintenance of these cells. This gap in our knowledge is, in part, due to the lack of a consensus about the surface phenotype of thymic macrophages. Various groups have used different markers to identify these cells, such as F4/80 and Mac-3 (LAMP-2)[31], or CD4 and CD11b[32], or Mac-2 (galectin 3), F4/80 and ED-1 (CD68)[33]. Most commonly, researchers employ F4/80 and CD11b[3437]. However, none of these markers is macrophage-specific: F4/80 is also 
expressed on eosinophils and monocytes[38,39], while CD11b is present on most myeloid cells. The lack of a clear phenotypic definition of thymic macrophages has translated into the absence of models that target genes specifically in this population. For example, although macrophages in various

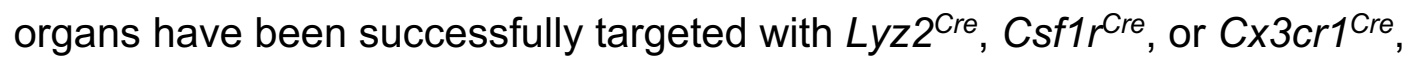
very few studies have used these models in the thymus[37,40,41].

Only a handful of studies has explored the origin of thymic macrophages. progenitors in the thymus based on an improved single cell in vitro culture and in vivo transplantation experiments[42,43]. However, these conclusions have been questioned based on fate-mapping experiments using $1 / 7 r^{C r e}$ that found very limited contribution of lymphoid progenitors to thymic macrophages in vivo in unperturbed mice[44]. Most recently, Tacke et al. used parabiosis to

104 rule out circulating monocytes as a major source for thymic macrophages[37].

105 The same study also performed fate-mapping experiments to show that most thymic macrophages descend from Flt3 ${ }^{+}$HSC-derived progenitors. However, the contribution of earlier waves of hematopoiesis has not been explored. other tissue-resident macrophages. We started by providing a clear definition

110 of thymic macrophages according to the guidelines set by the Immunological

111 Genome Consortium (IMMGEN)[38] and characterized their surface

112 phenotype and transcriptional signature. Using single-cell RNA sequencing

113 (scRNA-Seq), we identified two populations of thymic macrophages with

114 distinct localization. We explored the origin of these cells through genetic fate 115 mapping, shield chimeras, and embryonic thymus transplantation and 
documented that three separate waves of progenitors give rise to resident thymic macrophages. Altogether our work fills an important gap in our understanding of resident thymic macrophages and provides the framework for future functional characterization of these cells.

\section{Results:}

\section{CD64, F4/80, and MerTK identify the macrophages in the thymus}

To unambiguously and comprehensively identify macrophages in the thymus, we evaluated several of the prototypical macrophage markers, MerTK, CD64, and F4/80[38]. Only MerTK identified a population that stained completely with the other two markers (Fig. 1a). F4/80 labeled Siglec $\mathrm{F}^{+} \mathrm{SSC}^{\text {hi }}$ eosinophils and $\mathrm{CD} 4^{+} \mathrm{CD} 11 \mathrm{~b}^{+}$monocytes, in addition to MerTK ${ }^{+}$cells (Fig. S1a). CD64 identified CD11b+F4/80 lo monocytes (Fig. S1b). Thus, MerTK appeared to be the best marker for macrophages in the thymus. However, staining with MerTK and F4/80 was relatively dim even when the brightest fluorochromes (e.g., PE) were used and could not be resolved fully from the isotype control (Fig. S1c). For practical purposes, to identify macrophages, we used CD64 together with F4/80 and CD11b to exclude monocytes (Fig. S1d). In addition, we also employed forward scatter (cells size) to exclude cells that appeared $\mathrm{MerTK}^{+}, \mathrm{CD}^{+} 4^{+}, \mathrm{F} 4 / 80^{+}$, but were much smaller and less granular than the rest of the macrophages, and many of them were Thy $1^{+}$ (Fig. S1e).

\section{The $\mathrm{CD} 64^{+} \mathrm{F} 4 / 80^{+}$MerTK ${ }^{+} \mathrm{CD} 11 \mathrm{~b}^{\text {lo }} \mathrm{FSC}{ }^{\text {hi }}$ cells had typical macrophage} morphology with abundant cytoplasm (Fig. 1b). These cells did not express lineage markers characteristic of T cells (CD3e), B cells (CD19), eosinophils 
141 (Siglec F), NK cells (NK1.1), neutrophils ( $\mathrm{Gr} 1)$, or plasmacytoid dendritic cells

142 (Siglec H) (Fig. 1c). However, they expressed phagocytic receptors such as

143 TIM4, CD51, and Axl (Fig. 1d).

144 To ascertain that our gating strategy identifies all macrophages in the

145 thymus in an unbiased way, we performed scRNA-Seq of sorted Csf1rGFP+

146 and $C d 11 c^{Y F P+}$ thymic cells. Csf1r is required for the survival of most

147 macrophages and is considered their definitive marker[45,46], while $C d 11 c^{\text {YFP }}$

148 is expressed in many myeloid cells, including macrophages[47]. Both

149 reporters identified an overlapping set of cells (Fig. S2a). Two clusters

150 expressed the macrophage/monocytes-specific transcription factor Mafb and

151 high levels of Fcrg1 (CD64), Mertk, and Adgre1 (F4/80), indicating that they

152 are macrophages (Fig. 1e). An additional cluster expressed Mafb together

153 with Fcgr1 and Adgre1 but not Mertk, fitting the description of monocytes.

154 Altogether the scRNA-Seq data confirmed that our flow cytometry gating had

155 identified all macrophages in the thymus. Importantly, MerTK ${ }^{+}$cells could not

156 be labeled by intravenously injected CD45 antibody (Fig. 1f), proving that they

157 reside in the parenchyma of the organs and not in the blood vessels. Based

158 on the above data, we will refer to $\mathrm{CD} 64^{+} \mathrm{F} 4 / 80^{+} \mathrm{MerTK}^{+} \mathrm{CD} 11 \mathrm{~b}^{\text {lo }} \mathrm{FSC} \mathrm{C}^{\text {hi }}$ cells as

159 thymic macrophages. The smaller $\mathrm{CD} 64^{+} \mathrm{F} 4 / 80^{\mathrm{lo}} \mathrm{CD} 11 \mathrm{~b}^{+} \mathrm{FSC} \mathrm{C}^{\mathrm{hi}}$ population did

160 not express MerTK, but most of them expressed Ly6C, and we classify them

161 as thymic monocytes.

162 Thymic macrophages expressed CD11c, MHC2, and SIRP $\alpha$ that made

163 them partially overlap with $\mathrm{CD} 11 \mathrm{c}^{+} \mathrm{MHC}^{+}$classical dendritic cells (cDCs),

164 thus making problematic the unambiguous identification of thymic cDCs

165 based only on these two markers (Fig. S1f). Proper identification of cDC in the 
166

167

168

169

170

171

172

173

thymus requires the exclusion of macrophages based on CD64 or MerTK

staining. Otherwise, the cDCs, particularly the SIRP $\alpha^{+} \mathrm{cDC} 2$ subset, would be contaminated with macrophages that account for $\sim 25 \%$ of cDC2 (Fig.

S1g).

Thymic macrophages were $\sim 0.1 \%$ of all the cells in the thymus of young adult mice and numbered $\sim 4 \times 10^{5}$ on average per mouse (Fig. $1 \mathrm{~g}$ ). We did not find statistically significant differences in their percentages between 4 and 11 weeks of age. Still, there was a significant decline in their numbers with age, consistent with the beginning of thymic involution (Fig. 1h).

To determine if thymic macrophages are avid phagocytes, we evaluated their participation in the phagocytosis of apoptotic cells in the thymus by TUNEL staining. We used TIM4 and MerTK as single stains to identify macrophages in tissue sections because they are not expressed on any other cell type in the thymus instead of following our multiple-label flow cytometry strategy. Most of the TUNEL ${ }^{+}$cells could be found clearly inside or closely associated with MerTK ${ }^{+}$and $\mathrm{TIM}^{+}$cells in the thymus (Fig. S3a and b). On average, $\sim 85 \%$ of all TUNEL ${ }^{+}$cells were within $5 \mu \mathrm{m}$ of $\mathrm{MerTK}^{+}$cells, indicating that thymic macrophages are the major phagocytic population in the thymus (Fig. S3c). The degree of co-localization between TUNEL ${ }^{+}$cells and $\mathrm{TIM}^{+}$cells was slightly lower, $\sim 75 \%$ on average, possibly reflecting the absence of TIM4 expression on a small proportion of thymic macrophages (Fig. 1d). 

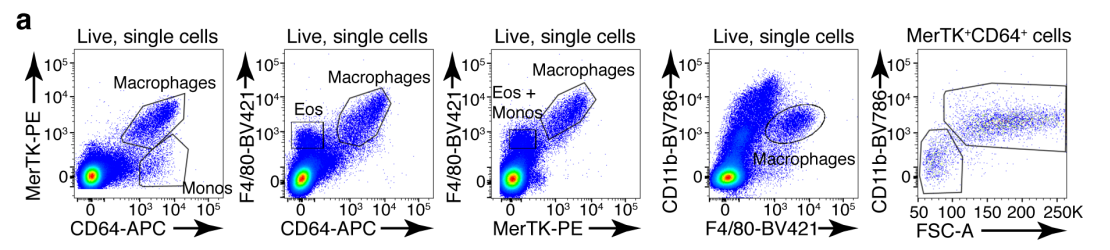

b

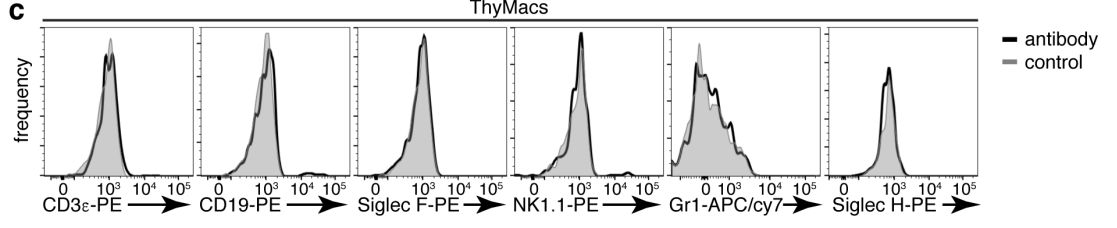

d
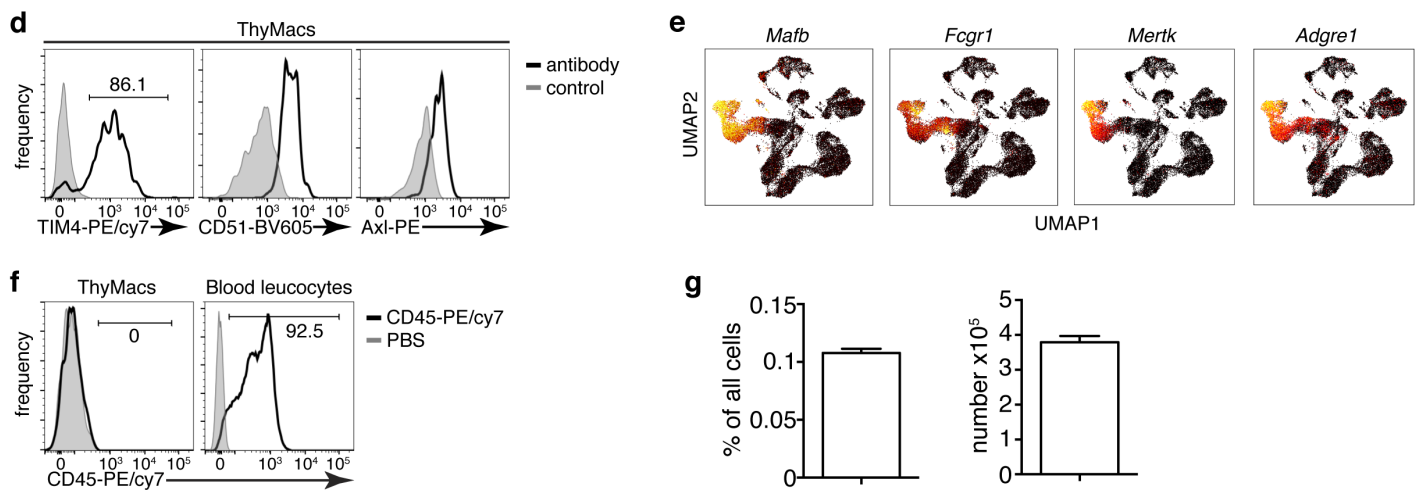

g
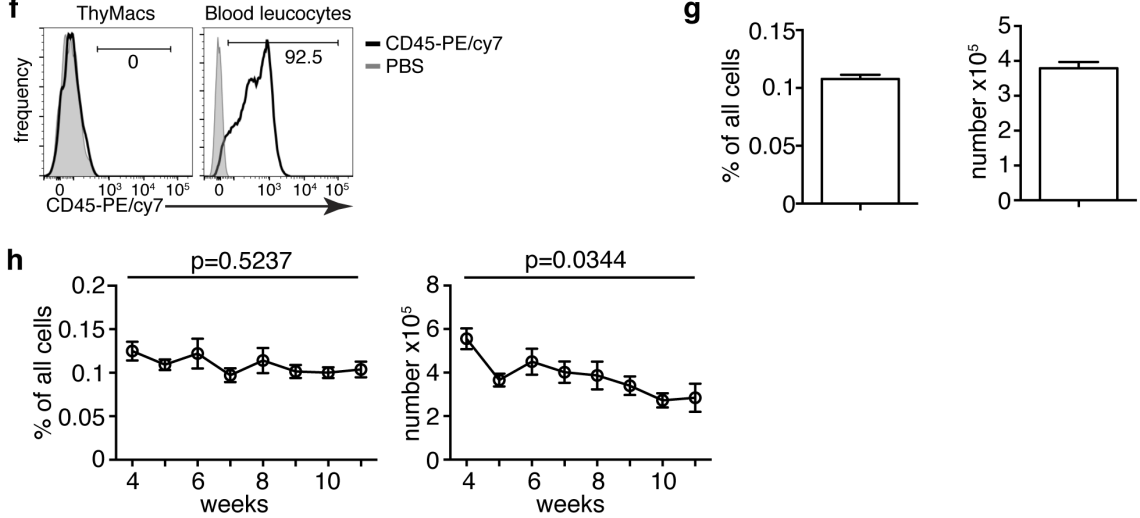

Figure 1. Thymic macrophages (ThyMacs) can be identified by the

expression of CD64, MerTK and F4/80. a Flow cytometric analysis of

enzymatically digested thymus tissue with macrophage markers CD64,

MerTK, F4/80, and CD11b. More details about the confirmation of the

identities of eosinophils and monocytes can be found in Fig. S1a and b. b

Pappenheim (Hemacolor Rapid staining kit) staining of sorted ThyMacs. c

Lack of expression of lineage markers associated with other cell types on

197 ThyMacs. d The expression on ThyMacs of three receptors for phosphatidylserine that participate in the phagocytosis of apoptotic cells. e scRNA-Seq data showing the expression of prototypical macrophage genes Mafb, Fcgr1 (CD64), Mertk, and Adgre1 (F4/80) among thymus cells sorted as $C s f 1 r^{G F P+}$ and $C d 11 c^{Y F P+}$. $\mathbf{f}$ Labeling of ThyMacs with intravenously 
injected anti-CD45-PE antibody or PBS. The labeling of blood leucocytes is

203 shown for comparison. g Average numbers and percentages of ThyMacs in 4-

20411 weeks old mice, $\mathrm{n}=82$. $\mathbf{h}$ Comparison of the numbers and percentages of

205 ThyMacs in mice of different ages, $n=82$. All flow cytometry plots are

206 representative of at least 3 independent repeats. The numbers in the flow

207 cytometry plots are the percent of cells in the respective gates. Data in $\mathbf{g}$ and

$208 \mathbf{h}$ represent mean \pm SEM. Statistical significance in $\mathbf{h}$ was determined with one209 way ANOVA.

\section{Transcriptional signature of thymic macrophages}

To further understand the identity and functions of the thymic

macrophages, we sorted and subjected them to RNA sequencing analysis as

214 part of the IMMGEN's Open Source Mononuclear Phagocyte profiling.

Thymic macrophages displayed a transcription profile consistent with tissue-

resident macrophages and did not express genes characteristic of other

217 lineages (Fig. 2a). Then, we examined the expression of the core signature macrophage genes[38] and found that they were enriched in thymic macrophages but not in Sirpa ${ }^{+}$or $X_{c r 1^{+}}$thymic cDCs (Fig. 2b). On the contrary, cDC core signature genes were abundantly expressed in both

221 thymic cDC subsets but not in thymic macrophages. These findings establish

222 that although thymic macrophages and cDCs share the thymic

223 microenvironment and expression of CD11c and $\mathrm{MHC} 2$, they have distinct 224 transcriptional profiles.

225 We then compared the gene expression profile of thymic macrophages to 226 that of other well-characterized macrophage populations available from 
IMMGEN. Because of the abundance of samples, we limited our comparison

228 to only nine types of tissue-resident macrophages under steady-state

229 conditions - splenic red pulp macrophages, Kupffer cells, broncho-alveolar

230 lavage macrophages, large peritoneal cavity macrophages, white adipose

231 tissue macrophages, aorta macrophages, central nervous system microglia,

232 and spinal cord microglia. Hierarchical clustering revealed that thymic

233 macrophages were most closely related to splenic red pulp macrophages and

234 Kupffer cells (Fig. 2c).

235 To better identify the unique functions of thymic macrophages, we looked

236 for genes that were differentially expressed in these cells compared to other

237 tissue-resident macrophages. We set three criteria: 1) high expression in

238 thymic macrophages ( $>500) ; 2)>5$ fold higher expression than the average

239 value in the nine populations of non-thymic macrophages; 3) expression in

240 thymic macrophages is higher than any non-thymic macrophage samples. A

241 total of 44 genes met these criteria, and we consider them to constitute the

242 transcriptional signature of thymic macrophages (Fig. 2d). These included

243 several degradation enzymes and their inhibitors (Cst7, Mmp2, Mmp14,

244 Dnase1/3, Serpina3g, Acp5), non-classical MHC molecules (H2-M2, H2-Q6,

245 H2-Q7), metabolic enzymes (Chst2, Ass1, Kynu, Cp, Dgat2, Sorl1, Lap3),

246 molecules involved in innate immunity (Ifit2, I/18bp, Mefv, Lgals3bp) and

247 extracellular signaling molecules and their receptors (Pdgfa, Cxcl16, I/2rg,

248 Gpr157). We also looked for transcription factors (TFs) highly expressed in

249 thymic macrophages and could potentially regulate critical gene networks in

250 these cells. A total of 25 TFs were highly expressed in thymic macrophages

251 (>250) and were at least 2-fold higher as compared to the non-thymic 
macrophages (Table S1). Among them were several TFs involved in type I

253 interferon (IFN-I) signaling (Stat1, Stat2, Irf7, and Irf8) and lipid metabolism

254 (Nr1h3, Pparg, Srebf1, and Rxra) (Fig. 2e). Notably, Runx3 that is important

255 for the development and function of cytotoxic T lymphocytes[48], innate

256 lymphoid cells[49], and Langerhans cells[50] was highly expressed in thymic

257 macrophages. SpiC that has well-documented roles in the development of red

258 pulp macrophages in the spleen, and bone marrow macrophages[51,52] was

259 also highly expressed in thymic macrophages, further strengthening the

260 argument for the similarity between thymus, spleen and liver macrophages.

261 To confirm the expression of Spic in thymic macrophages, we analyzed the

262 thymus of Spic ${ }^{G F P}$ mice[52]. We found that all Spic ${ }^{G F P+}$ cells were

263 macrophages (Fig. S4a and b), making them the most specific thymic

264 macrophage reporter strain compared with $L y z 2^{G F P}$, MAFIA (Csf1 $\left.r^{G F P}\right)$,

$265 C d 11 c^{Y F P}$, and $C \times 3 c r 1^{G F P}$ mice (Fig. S4c-e). However, only $\sim 80 \%$ of thymic

266 macrophages were Spic ${ }^{G F P+}$ suggesting heterogeneity within the cells (Fig.

267 S4f and g).

268 Several dominant pathways emerged when we grouped the 500 most

269 highly expressed genes in thymic macrophages according to gene ontology

270 (GO) terms (Fig. 2f). It was notable that five of the ten most highly enriched

271 GO pathways concerned antigen presentation of both exogenous and

272 endogenous antigens. These data complement our flow cytometry findings of

273 expression of MHC2 and suggest that thymic macrophages could be potent

274 antigen-presenting cells and might play a role in negative selection or agonist

275 selection of thymocytes. Two other highly enriched GO pathways were

276 involved in lysosomal biogenesis and functions, highlighting the high capacity 
of these cells to degrade phagocytosed material. Thus, our transcriptional

279 that bear significant similarity to spleen and liver macrophages and are

280 specialized in lysosomal degradation of phagocytosed material and antigen

281 presentation.

a

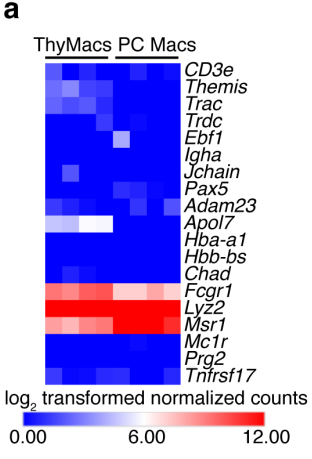

C

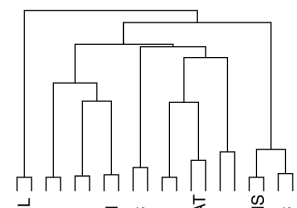

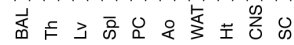

e

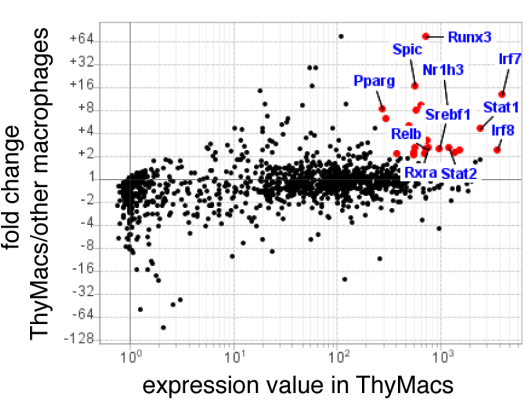

b

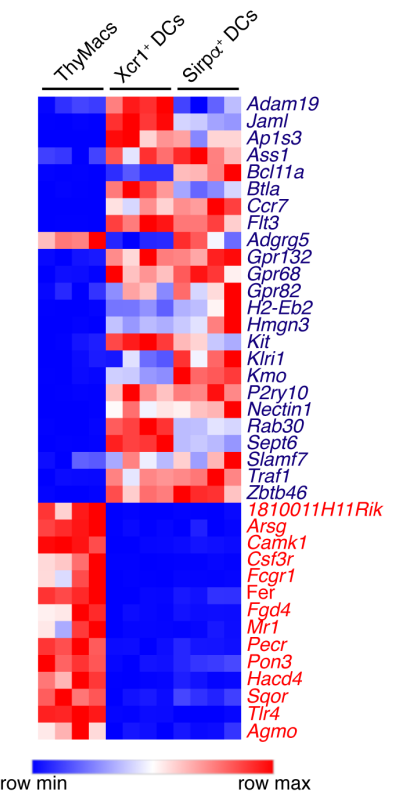

d

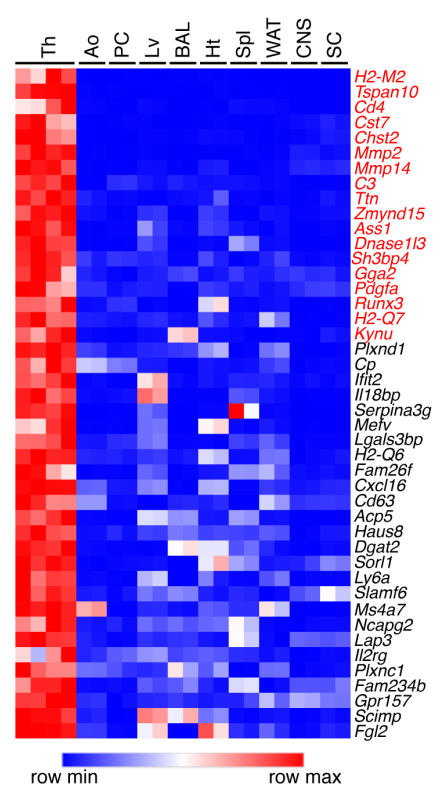

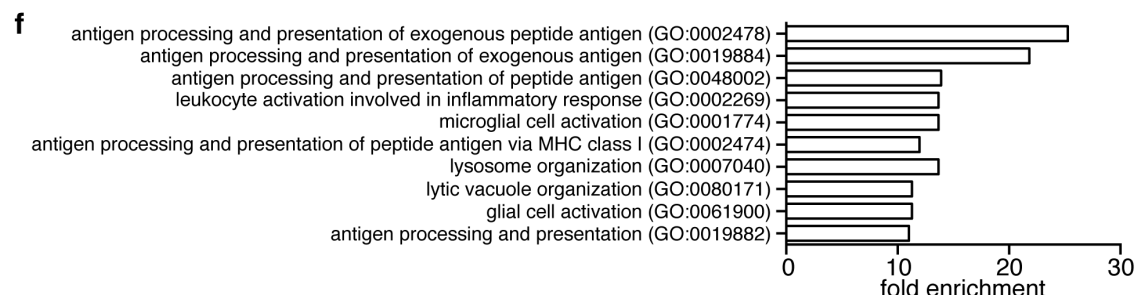

Figure 2. Transcriptional profile of thymic macrophages (ThyMacs). a

283 Expression of lineage-specific genes in ThyMacs and peritoneal cavity

284 macrophages (PC Macs). b Expression of cDC-specific genes (gene names in

285 blue) and macrophage-specific genes (gene names in red) in ThyMacs and two populations of thymic cDCs (ThyDCs) $-X_{c r 1^{+}}$ThyDCs and Sirpa ${ }^{+}$ 
ThyDCs. c Hierarchical clustering of ThyMacs and nine other populations of

288 tissue-resident macrophages in duplicates. d Highly expressed $(>500)$ genes

289 enriched (>5-fold) in ThyMacs (4 samples) compared to nine other tissue-

290 resident macrophage populations (two samples each). The genes in red are

$291>10$-fold up-regulated in thymic macrophages. e Comparison of the geometric

292 mean expression of transcription factors in thymic macrophages (4 samples)

293 and the nine other macrophage populations (2 samples each). Transcription

294 factors with expression $>250$ and fold change $>2$ are marked with red dots. $\mathbf{f}$

295 Top 10 GO pathways in ThyMacs based on the 500 most highly expressed

296 genes in these cells. Th - ThyMacs, Spl - spleen red pulp macrophages, Lv -

297 Kupffer cells, BAL - bronchoalveolar lavage macrophages, PC - peritoneal

298 cavity macrophages, Ao - aorta macrophages, $\mathrm{Ht}$ - heart macrophages, WAT

299 - white adipose tissue macrophages, CNS - central nervous system

300 microglia, SC - spinal cord microglia.

\section{Yolk-sac progenitors contribute to embryonic thymic macrophages}

The ontogeny of thymic macrophages has been examined by only one

304 study since the realization that many tissue-resident macrophages are

305 descendants from embryonic progenitors[37]. Based on Flt3 ${ }^{\text {Cre }}$ fate-mapping,

306 the authors concluded that most adult thymic macrophages derive from

307 HSCs. To determine if yolk-sac (YS) progenitors contribute to embryonic

308 thymic macrophages, we used $C \times 3 c r 1^{\text {CreER }}$ fate mapping[53]. Injection of 4-

309 OHT at E9.5 in ROSA26 $6^{\text {LSL-GFP }}$ mouse mated with a Cx3cr1 ${ }^{\text {CreER }}$ male

310 permanently tags YS progenitors and their descendants with GFP (Fig. 3a).

311 Indeed, E19.5 microglia that are exclusively derived from YS progenitors were 
312 labeled to a high degree (Fig. 3b). After adjusting for incomplete labeling

313 based on the microglia, we found that at E15.5 $>50 \%$ of thymic macrophages

314 were fate mapped, i. e. from YS origin (Fig. 3c). However, GFP ${ }^{+}$thymic

315 macrophages decreased to just $\sim 11 \%$ at E19.5, suggesting that $Y S$

316 progenitors establish the embryonic thymic macrophage pool but are quickly

317 replaced by subsequent wave(s) of fetal liver monocytes or HSC-derived

318 macrophages.

319 To pinpoint when do the HSC-derived progenitors enter the thymus, we

320 devised two complementary experiments. First, we evaluated the contribution

321 of circulating adult monocytes to thymic macrophages without the

322 confounding effect of radiation damage on the thymus. We created shield

323 chimeras by subjecting CD45.1 mice to a lethal dose of irradiation while

324 protecting their upper body and the thymus with a $5 \mathrm{~cm}$ lead shield followed

325 by reconstitution with CD45.2 bone marrow (Fig. 3d). While the donor-derived

326 monocytes in the blood were, on average $20 \%$, less than $2 \%$ of thymic

327 macrophages were CD45.2 $2^{+}$(Fig. $3 e$ and f), suggesting a relatively minor

$328(<10 \%)$ contribution of adult circulating monocytes to the thymic macrophage

329 pool consistent with a previous report[37]. Second, we transplanted E15.5

330 embryonic thymuses expressing GFP ubiquitously under the control of the

331 ROSA26 locus (ROSA26 ${ }^{G F P}$ ) under the kidney capsule of adult mice and

332 analyzed them six weeks later (Fig. $3 g$ ). By that time, $>99 \%$ of thymocytes

333 were derived from GFP- host HSCs (Fig. 3h and i). As a positive control for

334 donor-derived cells, we used the thymic epithelial cells. The vast majority of

$335 \mathrm{EpCAM}^{+}$thymic epithelial cells (>90\%) were still $\mathrm{GFP}^{+}$. An identical

336 proportion of thymic macrophages was also GFP ${ }^{+}$. The results from our 
transplantation experiments show that the progenitors of almost all thymic

resident thymic macrophages are derived from multiple waves of progenitors.

340 Initially, the thymus is settled by YS-derived progenitors that contribute

341 substantially to the thymic macrophage pool during the embryonic period. In

342 parallel with them, YS-independent progenitors infiltrate the thymus before

343 E15.5 and establish themselves as the dominant population before birth.

344 Adult HSC-derived monocytes contribute relatively little to the pool of thymic

345 resident macrophages in young adult mice.
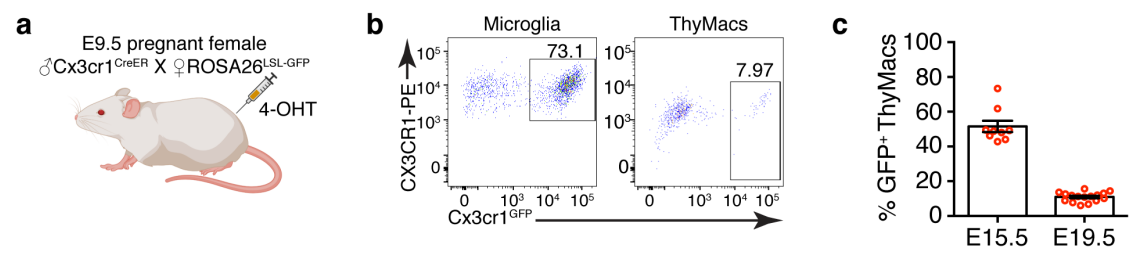

e
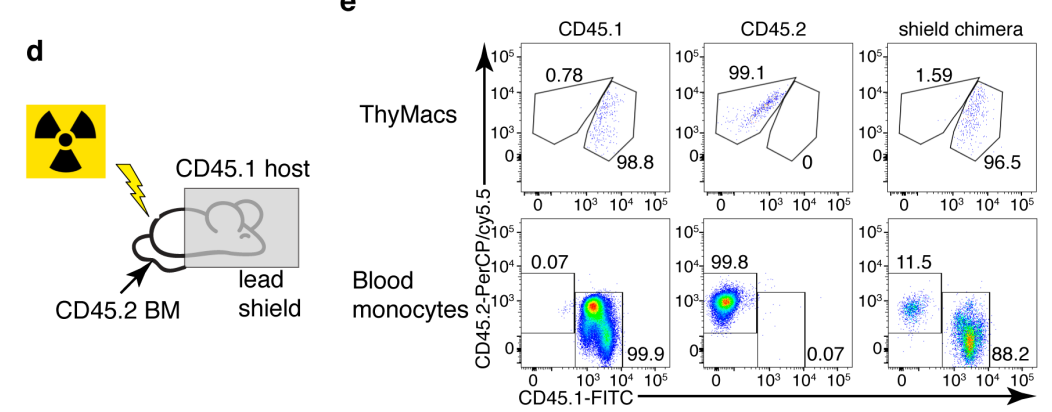

$\mathbf{f}$

h

G 15.5

E15.5
GFP $^{+}$thymus
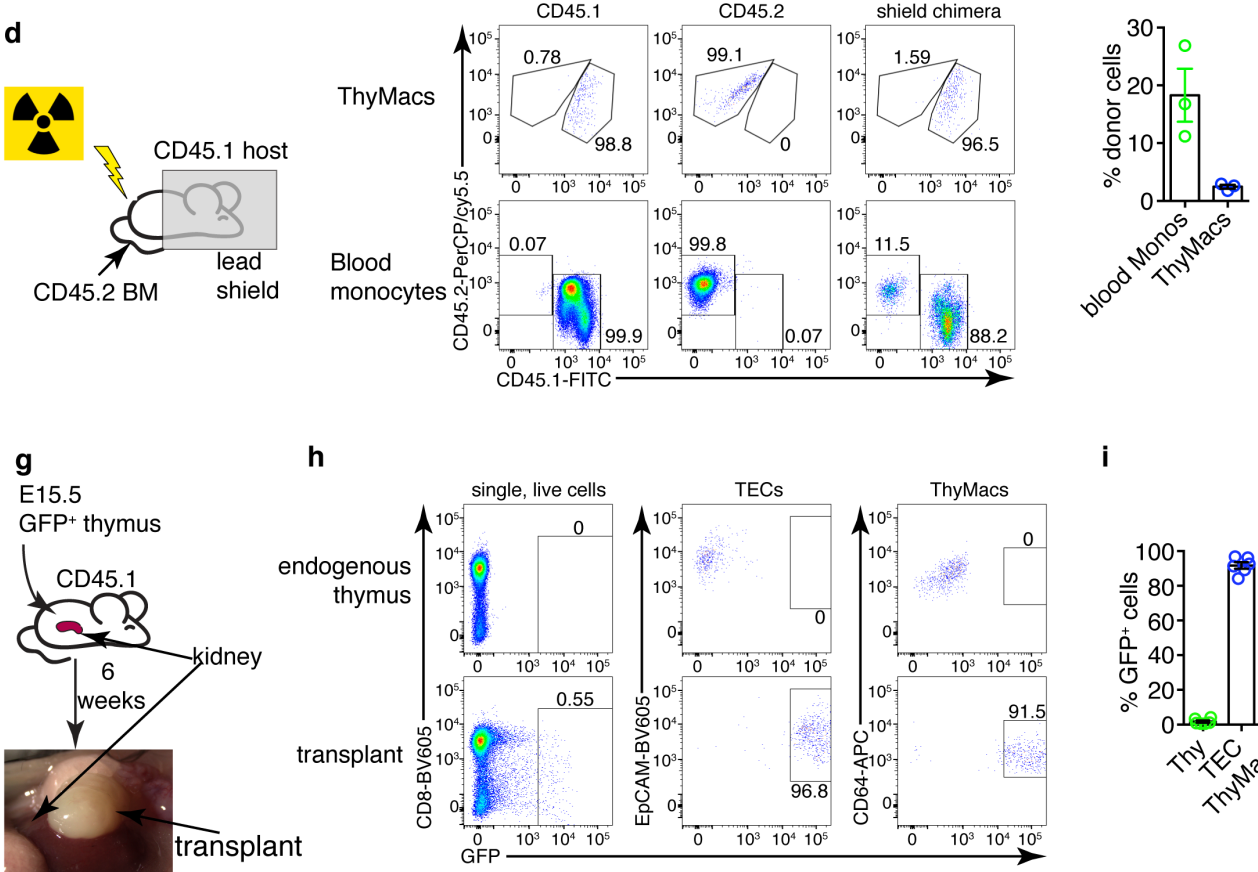

Figure 3. Yolk sac (YS)-derived and non-YS-derived embryonic

347 progenitors sequentially contribute to the thymic macrophage pool. a

348 Scheme of the YS-progenitor labeling experiments. E9.5 pregnant

ROSA26 ${ }^{L S L-G F P}$ mice mated with $C \times 3 c r 1^{\text {CreER }}$ males were injected with 4- 
351 flow cytometry plots of the $C \times 3 c r 1^{\text {GFP }}$ expression in microglia $\left(C D 45^{+} C D 11 b^{+}\right.$

352 cells in the brain) and ThyMacs of the pups. c Frequencies of GFP ${ }^{+}$ThyMacs

353 at E15.5 and E19.5 adjusted to the degree of labeling of microglia. $\mathbf{d}$ Scheme

354 of the shield chimera experiments. Congenic CD45.1 mice were lethally

355 irradiated with their upper body protected by a $5 \mathrm{~cm}$ thick lead shield and then

356 injected with CD45.2+ bone marrow. e Representative flow cytometric

357 analysis of ThyMacs and CD $115^{+} \mathrm{CD} 11 \mathrm{~b}^{+}$blood monocytes for donor-derived

$358\left(\right.$ CD45.2 $\left.{ }^{+}\right)$cells. Non-chimeric CD45.1 and CD45.2 samples serve as controls

359 for the gating. f Frequencies of donor-derived ThyMacs and blood

360 monocytes. $\mathbf{g}$ Scheme of the thymus transplantation experiments. $\mathbf{h}$

361 Representative flow cytometry plots of GFP expression in thymocytes (Thy),

$362 \mathrm{CD} \mathrm{CEpCAM}^{+}$thymic epithelial cells (TECs), and thymic macrophages

363 (ThyMacs) six weeks after the transplantation. The host thymus (endogenous

364 thymus) serves as a negative control. The percentages of $\mathrm{GFP}^{+}$cells are

365 indicated in the plots. i Frequencies of $\mathrm{GFP}^{+}$cells in different cell populations

366 in the transplanted thymus. Data in $\mathbf{c}, \mathbf{f}$, and $\mathbf{i}$ are mean $\pm S E M$ with two litters,

367 three, and five mice per group, respectively. The numbers in the flow

368 cytometry plots are the percent of cells in the respective gates. Each dot is an

369 individual mouse or embryo.

$371 \quad$ Thymic macrophages can proliferate in situ

372 The fact that macrophages can persist for many weeks in the thymus

373 without constant replacement from blood monocytes suggests that they can

374 divide in situ. Staining for the proliferation marker Ki-67 revealed that $\sim 4 \%$ of 
the cells expressed this marker compared to an isotype control (Fig. 4a and

b). To obtain further proof that thymic macrophages are proliferative, we thymic macrophages become $\mathrm{EdU}^{+}$with faster kinetics than thymocytes (Fig.

4c). The most likely explanation for this puzzling result is that some of the thymic macrophages have engulfed apoptotic thymocytes that have recently divided and incorporated EdU. Thus, EdU could have accumulated in these macrophages through phagocytosis and not through cell division. To

384 circumvent this caveat, we designed a pulse-chase experiment (Fig. 4d).

385 Mice were injected daily with EdU for 21 days so that all cells that proliferate in that period would incorporate the label. The vast majority of thymocytes and thymic macrophages became EdU ${ }^{+}$at d. 21 (Fig. 4e). After 21 more days of "chase period", only $\sim 0.2 \%$ of thymocytes had retained the EdU label, consistent with the existence of a very small population of long-term resident thymocytes consisting mainly of regulatory T cells and NKT cells[54] (Fig. 4e and f). However, $\sim 5 \%$ of the thymic macrophages were $\mathrm{EdU}^{+}$, suggesting

392 they divided during the labeling period. Finally, we sorted thymic 393 macrophages and subjected them to cell cycle analysis. Although almost all 394 thymic macrophages were in G0/G1 phase, a small population of $\sim 3 \%$ was in 395 G2/M phase of the cell cycle (Fig. $4 \mathrm{~g}$ and $\mathrm{h}$ ). Collectively, three independent approaches documented that $3-5 \%$ of thymic macrophages are actively

397 dividing under homeostatic conditions within the thymus. These findings can 398 explain the long-term maintenance of these cells within the organ without a 399 constant influx of progenitors. 
a

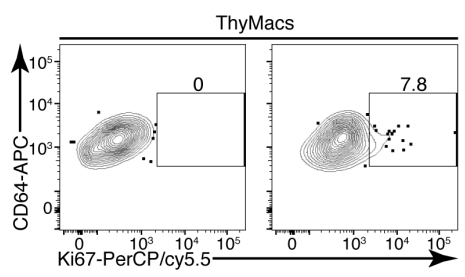

C

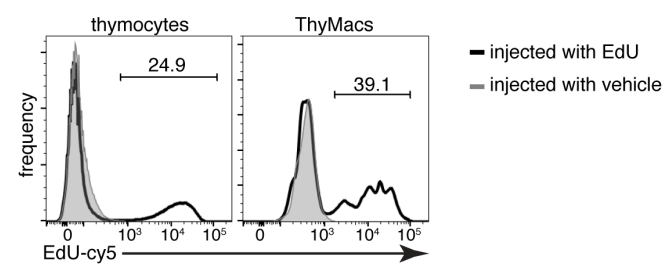

b

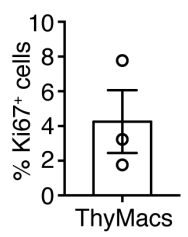

d

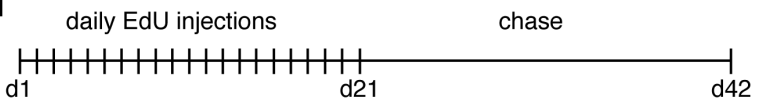

e

no EdU

21d pulse Thymocytes

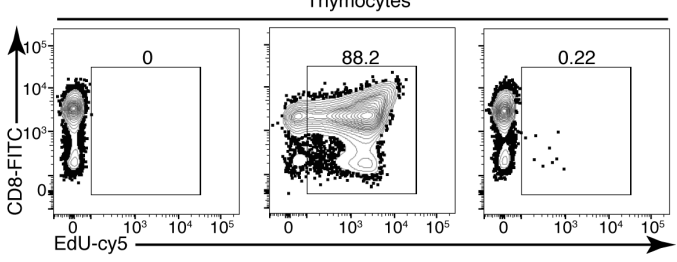

ThyMacs

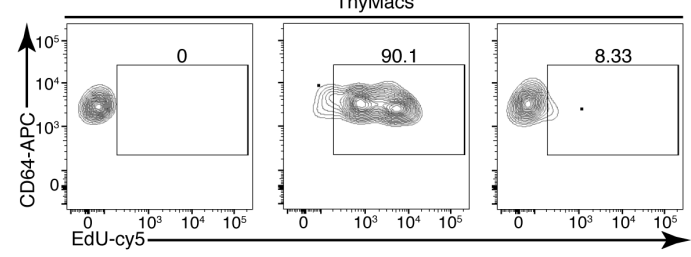

h

9
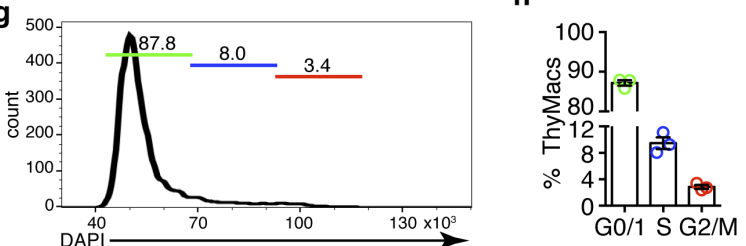
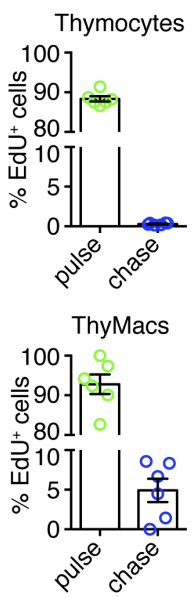

Figure 4. Thymic macrophages exhibit a low degree of proliferation. a

401 Example flow cytometry plots of Ki67 staining of thymic macrophages

402 (ThyMacs). b Frequency of Ki67 $7^{+}$thymic macrophages. c Example flow

cytometry plots of the EdU accumulation in thymocytes and thymic macrophages 2 hours after $1 \mathrm{mg}$ EdU i.p. or vehicle injection. The numbers

405 inside flow plots are the percentage of EdU ${ }^{+}$cells from mice injected with

406 EdU. Data are representative of three independent experiments. $\mathbf{d}$ Scheme of 407 EdU pulse/chase experiment: mice were injected daily with $1 \mathrm{mg}$ EdU i.p for 21 days and then rested for 21 more days. e Example flow cytometry plots of 
EdU staining of thymocytes (upper row) and ThyMacs (lower row). $\mathbf{f}$

410 Frequencies of EdU+ cells among thymocytes (top graph) and ThyMacs

411 (bottom graph). $\mathbf{g}$ Example flow cytometry plot of cell cycle analysis of FACS

412 sorted ThyMacs. $\mathbf{h}$ Frequencies of ThyMacs in different stages of the cell

413 cycle. The numbers in the flow cytometry plots are the percent of cells in the 414 respective gates. Data are mean \pm SEM from three mice (b and $\mathbf{h}$ ) or 6-7

415 individual mice (f). Each dot is an individual mouse.

\section{Expression of Timd4 and Cx3cr1 can distinguish two populations of}

\section{thymic macrophages}

419 To determine if thymic macrophages are heterogeneous, we turned to our scRNA-Seq data. Once we zoomed onto Mafb expressing cells, we could

421 distinguish three separate populations: 1) monocytes that expressed high

422 levels of Ly6c2 and Itgam (CD11b) but did not express Mertk; 2) Timd4+

423 (encoding TIM4) macrophages that also expressed high levels of Spic and Slc40a1, but low levels of $C \times 3 c r 1$; 3) $C \times 3 c r 1^{+}$macrophages that expressed

425 low levels of Timd4, Spic, and Slc40a1 (Fig. 5a and b). Both macrophages

426 and monocytes expressed Fcgr1 (CD64). A minority of macrophages

427 expressed high levels of Mki67, indicating that they might be proliferating,

428 corroborating our earlier data. Interestingly, most of the Mki67 expressing

429 macrophages belonged to the $\mathrm{C} \times 3 \mathrm{cr} 1^{+}$population.

430 We confirmed the results from scRNA-Seq by flow cytometry. We could

431 identify discrete $\mathrm{TIM}^{+} \mathrm{C} \times 3 \mathrm{Cr} 1^{\text {GFP- }}$ and TIM4 $4^{-} \mathrm{C} \times 3 \mathrm{cr} 1^{\text {GFP+ }}$ macrophages (Fig.

$4325 \mathrm{c})$. There was even $\mathrm{TIM} 4^{+} \mathrm{C} \times 3 \mathrm{cr} 1^{\mathrm{GFP+}}$ intermediate population that could not 433 be distinguished in the scRNA-Seq dataset, likely because of the lack of 
434 statistical power. To determine the localization of the two distinct macrophage

435 populations, we stained thymic sections from $C \times 3 c r 1^{\text {GFP }}$ mice with an antibody

436 to MerTK. The Cx3cr1 ${ }^{\text {GFP- }}$ MerTK $^{+}$cells correspond to Timd4 $4^{+}$macrophages,

437 while the $C \times 3 c r 1^{G F P+}$ MerTK $^{+}$cells would be the $C \times 3 c r 1^{G F P+}$ macrophages.

438 Strikingly, the two macrophage populations showed distinct localization.

$439 \mathrm{Timd}^{+}$macrophages were located in the cortex, while the $\mathrm{C} \times 3 \mathrm{cr} 1^{\mathrm{GFP}}$

440 macrophages resided in the medulla and the cortico-medullary junction (Fig.

$4415 d)$.

442 To better understand the differences between the two populations of thymic 443 macrophages, we looked for differentially expressed genes. We included the 444 thymic monocytes in the comparison, as these cells clustered the closest to

445 macrophages. Timd4 ${ }^{+}$macrophages expressed the highest levels of the

446 transcription factors Spic, Maf, and Nr1h3; the receptors for apoptotic cells

447 Axl, Mertk, and Timd4; and many Slc transporters such as Slc40a1, Slc1a3,

448 Slco2b1, S/c11a1, and Slc7a7 (Fig. 5e and Table S2). Cx3cr1 ${ }^{+}$macrophages

449 expressed high levels of the transcription factor Runx3; a distinct set of

450 phosphatidylserine receptors such as Stab1, Anxa5, and Anxa3; many

451 degradative enzymes such as Mmp2, Mmp14, Dnase1/3, Acp5, Lyz2, Ctsz,

452 Ctss, Ctsd, Ctsl; cytokines such as Pdgfa, Cxcl16, and Ccl12; and molecules

453 involved in MHC1 antigen presentation such as $B 2 m, H 2-M 2, H 2-K 1, H 2-Q 7$.

454 Thymic monocytes were characterized by differential expression of the typical

455 monocyte genes $L y 6 c 2$, Ccr2, and S100a4, and genes involved in MHC2

456 antigen presentation such as Ciita, $H 2-D M b 1, H 2-A b 1$, and $C d 74$. 
a

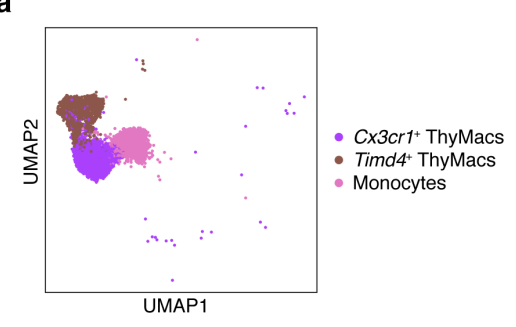

C

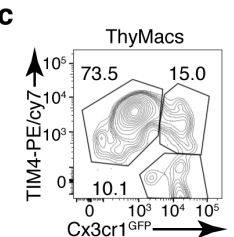

d

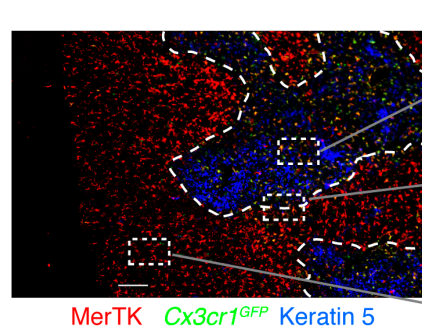

b
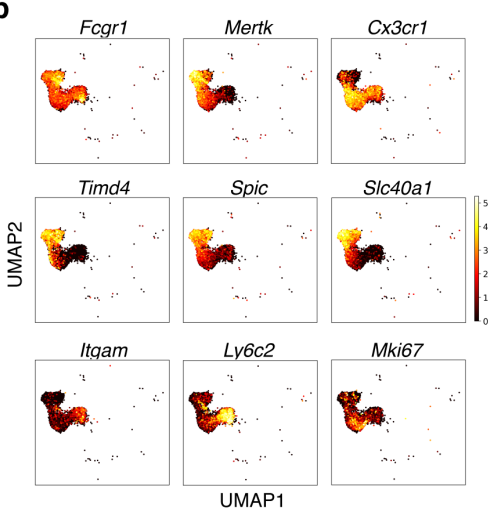

e

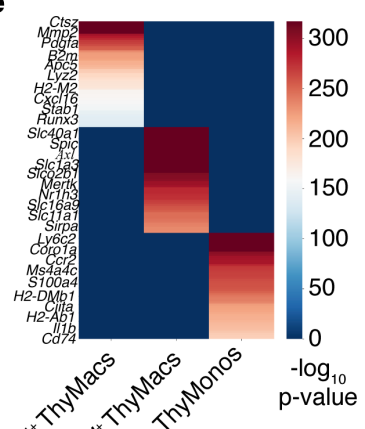

Figure 5: Two populations of macrophages with distinct localization

exist in the thymus. a UMAP clusters from Fig. S2 with high expression of the transcription factor Mafb fall into three groups: monocytes, Timd4 ${ }^{+}$ macrophages, and $\mathrm{C} \times 3 \mathrm{cr} 1^{+}$macrophages. $\mathbf{b}$ Expression of the indicated genes in the three Mafb-positive clusters. c A flow cytometry plot of $C \times 3 c r 1^{\text {GFP }}$ and TIM4 expression in ThyMacs. The plot is representative of $>10$ individual experiments. The numbers inside the plot are the percentages of the cell populations in the respective gates. $\mathbf{d}$ Immunofluorescent staining of the thymus of $C \times 3 c r 1^{G F P}$ mouse stained with MerTK (a marker for all macrophages) and Keratin 5 (a marker for medulla). The scale bar is $150 \mu \mathrm{m}$. Areas in the cortex, medulla, and the cortico-medullary junction (CMJ) represented by the dashed boxes are enlarged below to show the colocalization of $C \times 3 \mathrm{Cr}_{11}{ }^{\mathrm{GFP}}$ and MerTK signal in CMJ and medulla, but not in cortex. The scale bars in the images below are $20 \mu \mathrm{m}$. The images are 
representative of three individual mice. e Differentially expressed genes

473 among Timd4 $4^{+}$thymic macrophages, Cx3cr1 ${ }^{+}$thymic macrophages, and

474 thymic monocytes. The negative $\log _{10} p$-values for the genes expressed in

475 each cluster were calculated as described in the Materials and Methods, and

476 the top 50 differentially expressed genes were plotted in the figure. Ten of

477 these genes are listed on the left.

\section{$\mathrm{Cx}_{3 \mathrm{Cr} 1^{+}}$cells give rise to $\mathrm{Timd4}^{+}$cells during embryonic development}

To determine if the two populations of thymic macrophages are related, we

481 first analyzed the kinetics of their appearance during embryonic development.

482 At the earliest time point (E14.5), all thymic macrophages were $C \times 3 c r 1^{+}$, and

483 only $\sim 20 \%$ of them were also $\mathrm{TIM}^{+}$(Fig. 6a and b). The proportion of TIM4 ${ }^{+}$

484 cells increased at E17.5, and $\mathrm{TIM}^{+} \mathrm{C} \times 3 \mathrm{cr}^{-}$cells started to appear. In the

485 neonatal period, almost all macrophages were $\mathrm{TIM}^{+}$, and very few remained

486 TIM4- $^{-}$The proportion of TIM4- cells increased in 6 weeks old mice, but TIM4 ${ }^{+}$

487 macrophages remained the dominant population. These kinetics (Fig. 6c) are

488 consistent with $\mathrm{Timd}^{+}$macrophages developing from $\mathrm{C} \times 3 \mathrm{cr} 1^{+}$cells before

489 birth. Another plausible scenario is that distinct progenitors give rise to

490 different thymic macrophages populations (e.g., YS-progenitors give rise to

$491 \mathrm{C} 3 \mathrm{Cr}^{+}{ }^{+}$Timd4 $^{-}$and HSC-derived progenitors develop into Timd4 ${ }^{+}$

492 macrophages). To test the latter hypothesis, we re-visited the fate mapping of

493 YS progenitors results (Fig. 3a). Although a larger part ( 60\% at E15.5) of

$494 C \times 3 \mathrm{cr}^{+}{ }^{+}$TIM4- cells were derived from YS progenitors (Fig. 6d), a substantial

495 proportion ( $40 \%$ at E15.5) of $\mathrm{YS}$-derived $\mathrm{TIM}^{+}$macrophages could clearly

496 be identified at both E15.5 and E19.5, suggesting that YS progenitors can 
give rise to both $\mathrm{Cx} 3 \mathrm{Cr}^{+}$and $\mathrm{Timd}^{+}$cells. Extrapolating from the data, the

500 cells develop from $\mathrm{C} \times 3 \mathrm{cr} 1^{+}$cells during embryonic development. This

501 transition is complete in the first week after birth as there were essentially no $\mathrm{C} \times 3 \mathrm{cr} 1^{+}$thymic macrophages remaining at d.7 (Fig. 6a and b).

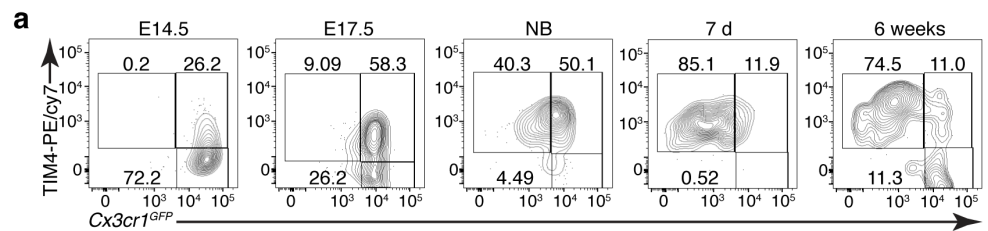

b

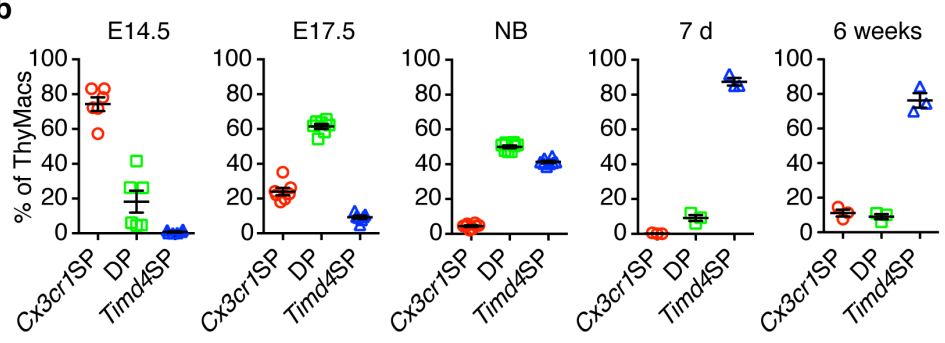

c

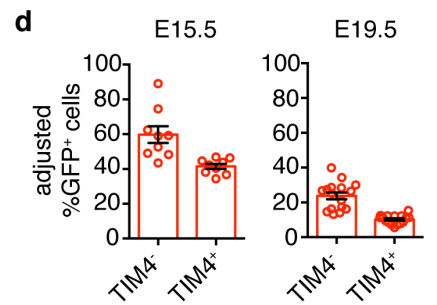

Figure 6. $\mathrm{Timd4}^{+}$thymic macrophages are derived from $\mathrm{C} 3 \mathrm{Cr1^{+ }}$ cells

505 during embryonic development. a Example flow cytometry plots for the expression of $\mathrm{Cx} 3 \mathrm{cr} 1^{\mathrm{GFP}}$ and TIM4 on thymic macrophages at different times during embryonic development (E14.5, E17.5), immediately after birth, at 7 days, and 6 weeks of age. b Frequencies of $\operatorname{Timd~}^{+} \mathrm{C} \times 3 \mathrm{cr} 1^{-}$(Timd4 singlepositive or Timd4SP), $\operatorname{Timd}^{+} \mathrm{C} \times 3 \mathrm{Cr} 1^{+}$(double-positive or DP), and

$510 \mathrm{C} \times 3 \mathrm{cr}^{+}{ }^{+}$Timd4- ( $\mathrm{C} \times 3 \mathrm{cr} 1$ single-positive or $\left.\mathrm{C} \times 3 \mathrm{cr} 1 \mathrm{SP}\right)$ thymic macrophages at

511 the indicated time points. c Kinetics of the changes in different subpopulations 512 of thymic macrophages from E14.5 to 6 weeks. d Frequencies at E15.5 and 
513 E19.5 of GFP-labeled cells among TIM4 $^{+}$or TIM4- $^{-}$cells in Cx3cr1 ${ }^{\text {CreER }}$ X

514 ROSA26 LSL-GFP embryos treated with 4-OHT at E9.5. Data is from at least two

515 independent experiments for each panel. Each symbol is an individual mouse

516 or embryo.

$\mathrm{Timd4}^{+}$thymic macrophages self-maintain, while $\mathrm{Cx} 3 \mathrm{Cr}^{+}$cells slowly

accumulate with age

To understand how are the resident thymic macrophage populations

521 maintained during adult life, we induced recombination in $C \times 3 c r 1^{C r e E R} X$

522 ROSA26 $6^{\text {LSL-GFP }}$ mice during the neonatal period (Fig. 7a) or at 6 weeks of age

523 (Fig. 7c) and compared the proportion of $\mathrm{GFP}^{+}$cells 3 and 42 days after

524 labeling. The extent of labeling of Timd4 $4^{+}$thymic macrophages did not

525 change within these 6 weeks, no matter whether the mice were treated with

526 Tamoxifen in the first week after birth or at 6 weeks (Fig. $7 b$ and d),

527 suggesting steady-state maintenance and absence of a significant influx from

528 unlabeled cells (e.g., monocytes). In contrast, the proportion of labeled

529 Timd4 $4^{-}$thymic macrophages decreased significantly 6 weeks after Tamoxifen

530 injection in both neonatal and adult mice, suggesting that this population was

531 being diluted by unlabeled cells. To further substantiate these findings, we

532 examined older WT mice and found out that the proportions of Timd4 thymic

533 macrophages increased with age, and in mice $>8$ months old, they accounted

534 for $\sim 70 \%$ of all macrophages in the organ (Fig. 7e). Thus, we conclude that

$535 \mathrm{Timd4}^{+}$macrophages can maintain themselves for long periods in the cortex

536 of the thymus, Since the first week of life, $C \times 3 c r 1^{+}$cells are slowly being

537 recruited to the medulla and cortico-medullary junction, and in aging mice 
form the predominant phagocytic population in the organ.

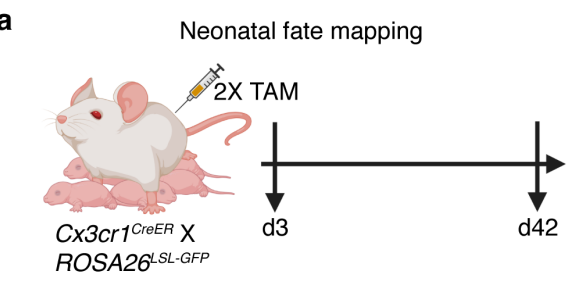

b

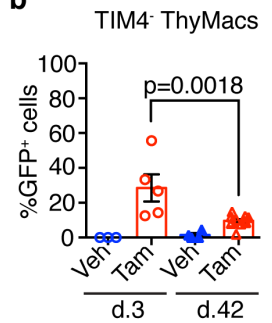

C
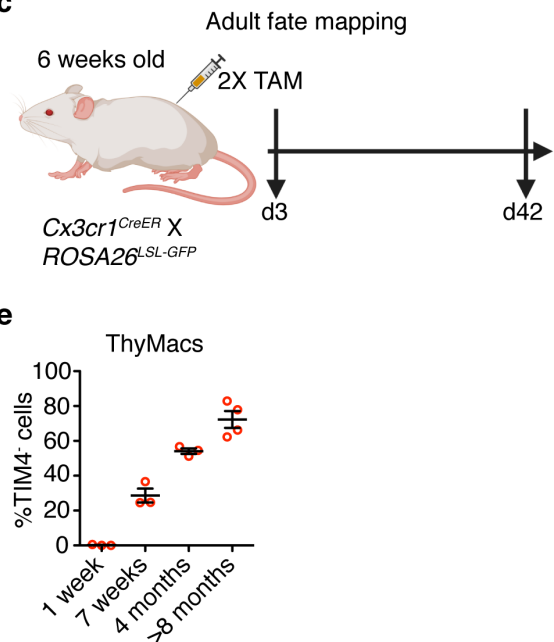

d

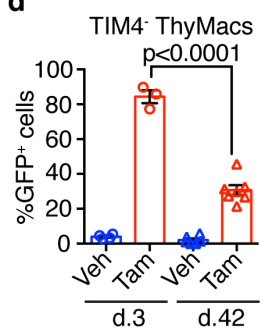

TIM4+ ThyMacs

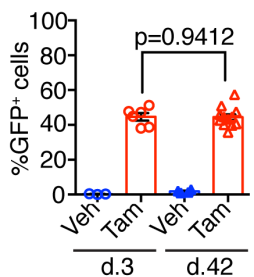

TIM4+ ThyMacs

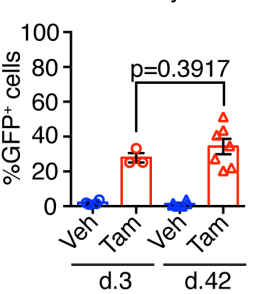

Figure 7. Timd4 ${ }^{+}$thymic macrophages self-maintain during adult life, while $\mathrm{C} \times 3 \mathrm{cr}^{+}$cells slowly accumulate with age. a Scheme of the neonatal fate mapping: A nursing dam was injected twice with Tamoxifen (Tam) or vehicle (Veh) in the first week after giving birth to $C \times 3 c r 1^{\text {CreER }} \times R O S A 26^{L S L-}$ GFP pups. Three or 42 days after the last injection, the pups were sacrificed, and the degree of labeling of Timd4 ${ }^{+}$and Timd4- thymic macrophages was examined by flow cytometry. b Frequencies of $\mathrm{GFP}^{+} \mathrm{Timd4}^{+}$or Timd4- thymic macrophages from neonatally fate mapped mice after 3 and 42 days. Vehicle injected nursing dam litters (Veh) served as a control for non-specific labeling. c Scheme of the adult fate mapping: Six weeks old $C \times 3 c r 1^{\text {CreER }} \times R O S A 26^{L S L-}$ GFP mice were injected twice with Tamoxifen (Tam) or vehicle (Veh). Three or 42 days after the last injection, the mice were sacrificed, and the degree of labeling of Timd4+ and Timd4- thymic macrophages was examined by flow 
cytometry. d Frequencies of $\mathrm{GFP}^{+}$Timd4 $^{+}$or Timd4- thymic macrophages

554 from adult fate mapped mice after 3 and 42 days. e Frequencies of TIM4-

555 thymic macrophages at different ages. The data is mean \pm SEM from 2

556 independent experiments (b) or at least 3 individual mice per time point (d and

557 e). Each symbol is an individual mouse. Statistical significance was

558 determined with unpaired Student's t-test.

\section{Discussion}

561 Here, we have described the phenotype, transcriptional profile, localization,

562 diversity, ontogeny, and maintenance of macrophages in the thymus. These

563 cells express the typical macrophage markers CD64, MerTK, and F4/80 and

564 are transcriptionally most similar to splenic red pulp macrophages and liver

565 Kupffer cells. However, they have a unique expression profile dominated by

566 genes involved in antigen presentation and lysosomal degradation. We found

567 that thymic macrophages consist of two populations with distinct localization.

$568 \mathrm{Timd}^{+}$macrophages occupied the cortex, while $\mathrm{Cx} 3 \mathrm{cr} 1^{+}$cells were located in

569 the medulla and the cortico-medullary junction. While YS-derived

570 macrophages dominated the early stages of thymus development, they were

571 quickly replaced by non-YS embryonic progenitors that gave rise to the

$572 \mathrm{Timd}^{+}$thymic macrophages that could proliferate and self-maintain. $\mathrm{C} \times 3 \mathrm{cr} 1^{+}$

573 macrophages started slowly accumulating after birth and in old mice became

574 the most abundant population.

575 Altogether our data depict thymic macrophages as typical tissue-resident

576 macrophages with origin from multiple hematopoietic waves, ability to self-

577 maintain, and expression of the core macrophage-specific genes. They were 
most similar transcriptionally to splenic red pulp macrophages and Kupffer efferocytosis and have efficient lysosomal degradation machinery. These

581 three populations also shared expression of the transcription factor Spic that

582 is induced by heme released following red blood cells phagocytosis[52].

583 However, the thymus is not known as a place for erythrocyte degradation.

584 Thus the mechanism for Spic up-regulation in thymic macrophages is unclear.

585 The unique features of thymic macrophages include high expression of 586 genes involved in the IFN-I pathway, antigen presentation, and lysosomal 587 degradation. The up-regulation of IFN-I-stimulated genes such as Stat1, 588 Stat2, Irf7, and Irf8 can be explained by the constitutive secretion of IFN-I by 589 thymic epithelial cells[55,56]. The purpose of IFN-I expression in the thymus 590 in the absence of a viral infection is unclear. Still one possibility is that it 591 mediates negative selection to IFN-dependent genes as part of central 592 tolerance.

593 Thymic macrophages highly express molecules involved in antigen 594 presentation, including $\mathrm{MHC} 1$ and $\mathrm{MHC} 2$, although the latter is expressed at 595 lower levels than in cDCs. Thus, they have the potential to present antigens 596 for both negative selection and agonist selection. These two activities have 597 traditionally been assigned solely to cDCs[57]. However, recent evidence 598 suggests that negative selection is most efficient when the cell that presents 599 the antigen to auto-reactive thymocytes is also the one that phagocytoses $600 \mathrm{it}[58]$. So, macrophages participation in thymocyte selection needs to be re601 evaluated with optimized isolation procedures and specific genetic tools. 
The extraordinary ability of thymic macrophages to engulf and degrade

603 apoptotic thymocytes has been appreciated for a long time[31], and our RNA-

604 Seq data provides additional supporting evidence for this function by

605 highlighting the up-regulation of pathways involved in lysosomal degradation.

606 An interesting topic for future research would be to understand how the metabolites derived from apoptotic cells are returned to the microenvironment to support the proliferation of immature thymocytes. A SoLute Carrier (SIc) genes-based program has been described in vitro[59], but its relevance to

610 tissue-resident macrophages remains to be determined. Altogether, our study

611 demonstrates that thymic macrophages are a unique subset of tissue-resident 612 macrophages and support the idea that resident macrophage phenotype is 613 determined by the combination of ontogeny, microenvironment, and other 614 factors[60].

615 Together with the study by Tacke et al., our work builds the following model 616 for thymic macrophage origin[37]: Thymic macrophages develop in three 617 distinct waves: YS-derived progenitors dominate the early stages of thymus 618 development but are replaced before birth by a second wave of YS-

619 independent embryonic progenitors that forms the bulk of thymic

620 macrophages after birth and can self-maintain into adulthood. With age, there

621 is a slow and steady influx of Timd4-Cx3cr1+ macrophage precursors that 622 occupy the medulla and cortico-medullary junction, becoming the major 623 phagocytic population in the thymus of older mice (>8 months). The second 624 wave of YS-independent macrophages is most likely the progeny of 625 embryonic HSCs based on Flt3 $3^{\text {Cre }}$ fate mapping that showed that $>80 \%$ of 626 thymic macrophages in adult mice are descendants of HSCs[37]. Whether 
HSC-independent fetal liver monocytes contribute to thymic macrophages and

628

650 with cortical thymic epithelial cells and die by neglect or are auto-reactive and

651 undergo clonal deletion in the cortex[61]. On the other hand, $C \times 3 \mathrm{cr}^{+}$ 
652

macrophages accumulate in the medulla; the thymic region specialized in negative selection to tissue-restricted antigens (TRA). They might contribute to the process in several ways: 1) by carrying TRAs from blood and peripheral organs. A similar process has been described for $\mathrm{CDC} 2\left(\mathrm{SIRP} \alpha^{+} \mathrm{DCs}\right)[62]$. In fact, $\mathrm{C} \times 3 \mathrm{Cr} 1^{+}$thymic macrophages could have contributed to this role because they were not distinguished from $\mathrm{cDC} 2$ in this study. 2) By capturing TRAs from Aire $^{+}$medullary thymic epithelial cells and presenting them to autoreactive thymocytes as shown for DCs[63-65]. 3) By phagocytosing apoptotic TRA-specific medullary thymocytes, a process we have observed before[58]. The exact involvement of thymic macrophages in the selection events in the thymus remains to be determined.

The accumulation of the $C \times 3 c r 1^{+}$cells in older mice has clear implications for thymus aging. One key feature of thymus involution is the accumulation of extracellular matrix produced by fibroblasts and the emergence of white adipocytes[66]. The $C \times 3 c r 1^{+}$subset is the predominant producer of the growth factor PDGF $\alpha$ that is required for the maintenance of adipocyte stem cells and can stimulate tissue fibrosis $[67,68]$. The gradual accumulation of $C \times 3 c r 1^{+}$ macrophages could increase the availability PDGF $\alpha$ in the aging thymus stimulating extracellular matrix production and differentiation of precursors into adipocytes. This model predicts that limiting the influx of $C \times 3 c r 1^{+}$ macrophage precursors could delay thymus involution.

Recent work described a novel phagocytic and antigen-presenting cell type in the thymus called monocyte-derived DCs[65]. The phenotype of these cells overlaps with the $\mathrm{CD} 64^{+} \mathrm{F} 4 / 80^{\mathrm{lo}} \mathrm{CD} 11 \mathrm{~b}^{+}$cells in our study. However, we favor the classification of these cells as monocytes based on their expression of 
Mafb, CD64, and Ly6C (Fig. 5b) and lack of expression of the defining DC transcription factor Zbtb46 (Fig. S2c)[69]. As monocytes can differentiate into cDC2, particularly in the context of inflammation[70], the precise identity and the relationship of this population to thymic $\mathrm{CDC} 2$ remain to be established. In the past several years, scRNA-Seq has come to the forefront of biologists' efforts to disentangle the cellular diversity of tissues. Several comprehensive studies have included samples from mouse or human thymus[71-73]. However, in these studies, too few thymic macrophages were sampled to give meaningful clustering results. Efforts specifically targeting the thymus have provided considerably more information[74,75], but macrophage diversity was still not recognized. Characterization of rare populations such as thymic macrophages ( $0.1 \%$ of all cells in the thymus) requires optimized enzymatic digestion procedures and enrichment strategies, as has been demonstrated already for thymic epithelial cells[76,77]. Our scRNA-Seq dataset provides a rich resource for the unbiased characterization of myeloid cells in the thymus and will greatly aid in the understanding of the myeloid landscape of the thymus.

In summary, our work comprehensively characterizes macrophages in the thymus and paves the way for exploration of their functions.

\section{Materials and methods}

\section{Mice}

$$
\text { C57BL/6Narl (CD45.2) mice were purchased from the National Laboratory }
$$
Animal Center, Taipei, Taiwan. MAFIA (MAcrophage Fas-Induced Apoptosis) [78], $C \times 3 c r 1^{\text {GFP }}$ [79], Spic ${ }^{\text {GFP }}$ [52], Cx3cr1 ${ }^{\text {CreER }}$ [53], and B6.SJL-Ptprca 
Pepcb/BoyJ (CD45.1) mice were purchased from the Jackson Laboratories. $C d 11 c^{Y F P}[80]$ and $L y z 2^{G F P}$ [81] mice have been described. Mice ubiquitously expressing GFP from the ROSA26 locus were generated by breeding

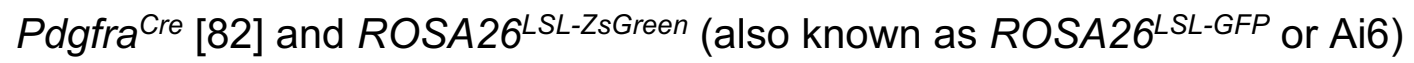
mice[83] (both from the Jackson Laboratories). A mouse from this cross was identified, in which the STOP cassette was deleted in the germline. It was designated ROSA26 ${ }^{G F P}$ and subsequently bred to C57BL/6 mice. All mice were used at 4-10 weeks of age unless otherwise specified. Mice were bred and maintained under specific pathogen-free conditions at the animal facility of National Yang-Ming University. All experimental procedures were approved by the Institutional Animal Care and Use Committee (IACUC) of National Yang-Ming University.

\section{Treatment with 5-Ethynyl-2'-deoxyuridine (EdU)}

Mice were i.p. injected with $1 \mathrm{mg}$ EdU (Carbosynth) dissolved in PBS daily for 21 days and then rested for 21 more days. Cells from the thymus were harvested on day 21 or 42 . In some experiments, the mice were sacrificed 2 hours after the first EdU injection.

\section{Shield chimera generation}

CD45.1 mice were anesthetized by i.p. injection of $120 \mu \mathrm{g} / \mathrm{g}$ body weight Ketamine hydrochloride (Toronto Research Chemicals) and $12 \mu \mathrm{g} / \mathrm{g}$ body weight Xylazine hydrochloride (Sigma). Anesthetized mice were taped to a 5 $\mathrm{cm}$ thick lead block so that the lead block covered the head and the chest down to the bottom of the rib cage. Then, they were irradiated with a lethal 
dose (1000 rad) from a ${ }^{137} \mathrm{Cs}$ source (Minishot II, AXR) so that only their

728 abdomen and hind legs were exposed. After recovery from anesthesia, the

729 mice were transfused i.v. with $10^{7}$ bone marrow cells from a congenic

730 (CD45.2) donor. Then, they were given Trimerin $(0.5 \mathrm{mg} / \mathrm{mL}$ Sulfadiazine +

$7310.1 \mathrm{mg} / \mathrm{mL}$ Trimethoprim, China Chemical and Pharmaceutical Co., Tainan,

732 Taiwan) in the drinking water for the first two weeks after the irradiation and

733 analyzed after six weeks.

734

\section{Cell isolation from thymus, blood, and peritoneal cavity}

Thymocytes were obtained by mechanical disruption of the thymus with a

syringe plunger. For myeloid cell isolation, mouse thymuses were cut into

mixture for thymic epithelial cell isolation. In some experiments, thymic

742 myeloid cells and thymic epithelial cells were enriched by $57 \%$ Percoll PLUS

743 (GE Healthcare) discontinuous gradient centrifugation at $4^{\circ} \mathrm{C}, 1800 \mathrm{rpm}$, for

74420 min without brake. Cells at the interface were collected and washed with

745 PBS to remove residual silica particles. Then the cells were resuspended in

746 PBS with $0.5 \%$ BSA (HM Biological), filtered through a $70 \mu \mathrm{m}$ filter, and kept

747 on ice.

749 Blood was isolated by cardiac puncture of sacrificed mice and immediately 750 diluted with PBS. After centrifugation, the cell suspensions were treated with 751 ammonium chloride-potassium lysis buffer for 3 min on ice once or twice. 
752

753

754

755

756

757

758

759

760

761

762

763

764

765

766

767

768

769

770

771

772

773

774

775

Peritoneal cavity cells were obtained by lavage with $5 \mathrm{~mL}$ PBS $+2 \mathrm{mM}$ EDTA (Merck). Following gentle massage, the cavity was opened with an abdominal incision, and lavage fluid was collected.

\section{Flow cytometry}

Single-cell suspensions $\left(0.5-2 \times 10^{6}\right.$ cells $)$ from thymus, blood, or

peritoneal cavity were blocked with supernatant from 2.4G2 hybridoma (a kind gift by Dr. Fang Liao, Academia Sinica, Taipei, Taiwan) and stained with fluorochrome- or biotin-labeled antibodies for 20 min on ice in PBS $+0.5 \%$ BSA + $2 \mathrm{mM}$ EDTA $+0.1 \% \mathrm{NaN}_{3}$ (FACS buffer). The following antibodies were used: CD11b (clone M1/70), MHC2 (M5/114.15.2), CD11c (N418), F4/80 (BM8), CD115 (AFS98), SIRP $\alpha$ (P84), CD45 (30-F11), NK1.1 (PK136), TIM4 (RMT4-54), Gr-1 (RB6-8C5), CD64 (X54-5/7.1), Siglec H (551), Ly6C (HK1.4), CD3 $\varepsilon$ (145-2C11), CD8 $\alpha$ (53-6.7), CD19 (6D5), B220 (RA3-6B2), CD4 (GK1.5), CD51 (RMV-7), CD45.1 (A20), CD45.2 (104), CX3CR1 (SA011F11), and EpCAM (G8.8) from BioLegend; AxI (MAXL8DS), MerTK (DS5MMER), and Ki67 (SolA15) were from eBioscience; Siglec F (E50-2440), CD90.2 (30-H12), and CD11c (HL3) were from BD Biosciences. Cells were washed, and if necessary, incubated for 20 more min with fluorochromelabeled Streptavidin: Streptavidin-AF647 (Jackson Immunoresearch) or Streptavidin-APC/cy7, Streptavidin-BV421, Streptavidin-BV605 (BioLegend). After the last wash, the cells were resuspended in FACS buffer containing DAPI (BioLegend), Propidium lodide (Sigma), or DRAQ7 (BioLegend) and analyzed immediately on an LSR Fortessa flow cytometer running Diva 8 
software (BD Biosciences). Typically, 500,000 cells were collected from

thymus samples. Data were analyzed using FlowJo software (TreeStar).

For intracellular staining, after surface antibody staining, the cells were

780 labeled with Zombie Aqua (BioLegend) for $30 \mathrm{~min}$ in ice. Then, the cells were

781 fixed with 2\% paraformaldehyde (Electron Microscope Sciences) in PBS for

78220 min on ice, permeabilized with either 0.5\% Triton-X 100 (Sigma) for 20 min

783 on ice, or with Foxp3 staining kit (eBioscience) according to the protocol

784 provided by the manufacturer, and stained with antibodies for intracellular

785 markers for $40-60$ min on ice.

786

For cell cycle analysis, $1-5 \times 10^{5}$ sorted thymic macrophages were fixed with $70 \%$ ethanol for $2 \mathrm{~h}$ on ice. The cells were spun down at $1800 \mathrm{rpm}$ for $20 \mathrm{~min}$ at $4^{\circ} \mathrm{C}$, washed with PBS, and stained with $1 \mu \mathrm{g} / \mathrm{ml}$ DAPI (BioLegend) for 30 min at room temperature in the dark. permeabilized with $0.5 \%$ Triton X-100 in PBS at room temperature for 20 min.

795 EdU was detected by adding an equal volume of $2 \mathrm{X}$ Click reaction buffer 796 consisting of $200 \mathrm{mM}$ Tris, $200 \mathrm{mM}$ ascorbic acid (Acros), $8 \mathrm{mM} \mathrm{CuSO}_{4}$ 797 (Acros), $8 \mu \mathrm{M}$ Cy5-azide (Lumiprobe) to the permeabilized cells resuspended 798 in $0.5 \%$ Triton X-100 in PBS and incubation at room temperature for $30 \mathrm{~min}$. 799 Cells were washed twice with $0.5 \%$ Triton X-100 in PBS and analyzed on a 800 flow cytometer. 


\section{Cell sorting}

803 The sorting of thymic macrophages was done following the IMMGEN

804 guidelines. Briefly, the thymuses of 3 male C57BL/6Narl mice were harvested

805 in ice-cold staining buffer containing phenol red-free DMEM (Gibco) with 10

806 mM HEPES (Sigma), 0.1\% $\mathrm{NaN}_{3}$, and 2\% FBS (Gibco). Single-cell

807 suspensions were prepared as described in the Flow cytometry section.

808 Percoll PLUS was used to enrich mononuclear cells. The cells were

809 resuspended at $10^{8} / \mathrm{mL}$ in staining buffer and labeled with appropriate

810 antibodies for 15 min in ice. To sort thymic macrophages, the cells were first

811 labeled with biotinylated antibodies to lineage markers (Lin) - CD3, CD8, Gr1,

812 B220. After washing, the cells were stained with antibodies to CD11b, F4/80,

813 CD45, CD64, and Streptavidin-APC/cy7 for 15 min in ice. For sorting thymus

$814 \mathrm{XCR}^{+}$and SIRP $\alpha^{+} \mathrm{cDC}$, antibodies to XCR1, SIRP $\alpha, \mathrm{CD} 11 \mathrm{c}, \mathrm{MHC} 2, \mathrm{CD} 64$,

815 and F4/80 were used. For sorting peritoneal cavity macrophages, antibodies

816 to ICAM2 and F4/80 were used. Immediately before sorting, the dead cells

817 were excluded with DRAQ7 or PI. For RNA Sequencing experiments, the

818 cells were double-sorted on FACS Melody, or Aria cell sorters (BD

819 Biosciences) and 1000 cells were directly deposited in TCL buffer (Qiagen),

820 frozen in dry ice and sent to IMMGEN for RNA sequencing. Four biological

821 replicates were prepared. For cytospin and cell cycle analysis, $1-5 \times 10^{5}$ cells

822 sorted on FACS Melody were collected in staining buffer. 
Sorted cells were mounted on Superfrost PLUS slides (Thermo Scientific) using a Cytospin centrifuge (Cytospin 3, Shandon) for $5 \mathrm{~min}$ at $500 \mathrm{rpm}$. Cells were fixed with $2 \%$ paraformaldehyde for $10 \mathrm{~min}$ at room temperature and

828 stained with the Hemacolor Rapid Staining Kit (Merck Millipore). Images were 829 collected on BX61 upright microscope (Olympus) using 100X objective with 830 immersion oil and captured with a CCD camera. Images were then analyzed 831 and processed with ImageJ (NIH) and Adobe Photoshop 5.5 (Adobe).

\section{RNA sequencing analysis}

RNA sequencing was done at IMMGEN using Smart-seq2 protocol[84,85]

839 (https://software.broadinstitute.org/morpheus). Hierarchical clustering was

840 done with Cluster 3.0 and visualized with Java TreeView. Only genes with

841 SD>20 were used (10602 genes). The metric used was Pearson correlation

842 (uncentered), and the clustering method was average linking. Gene

843 expression of mouse transcription factors[87] was visualized in MultiplotStudio

844 of GenePattern[88]. GO enrichment was calculated and visualized in R by

845 using clusterProfiler[89].

847 Timed pregnancies and embryonic thymus analysis

848 To set up timed pregnancies, each male mouse $\left(C \times 3 c r 1^{C r e E R / C r e E R}\right.$, $849 C \times 3 c r 1^{\text {GFP/GFP }}$ or C57BL/6) and female mouse (ROSA26 ${ }^{\text {LSL-GFP/LSL-GFP }}$ or 
C57BL/6) were housed together in the same cage for one night and separated

851 on the next day, which we defined as embryonic day 0.5 (E0.5). Female mice

852 were assumed to be pregnant if their weight gain was over $2 \mathrm{~g}$ at E8.5[90].

853 Thymuses from E14.5 and E17.5 embryos, neonatal, 1-weeks-old pups, and

854 adult mice (older than 6-weeks-old) were harvested, mechanically dissociated 855 with plastic sticks in 1.5-mL centrifuge tubes, and enzymatically digested with $8560.2 \mathrm{mg} / \mathrm{mL}$ DNase I and $0.2 \mathrm{mg} / \mathrm{mL}$ collagenase $\mathrm{P}$ in complete DMEM for 20 857 min at $37^{\circ} \mathrm{C}$ with frequent agitation. The cells were resuspended in PBS with $8580.5 \%$ BSA, filtered through a $70 \mu \mathrm{m}$ filter, kept on ice, and used flow 859 cytometric analysis as described in the Flow Cytometry section.

\section{Genetic fate mapping - E9.5, neonatal and adult}

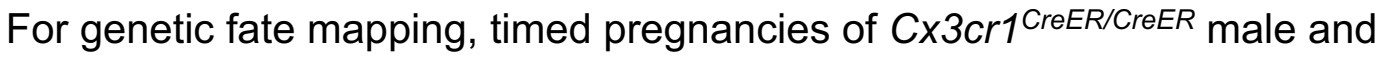
ROSA26 LSL-GFP/LSL-GFP female mice were set up as described. To label the

$864 \mathrm{C} \times 3 \mathrm{Cr}^{+}$erythromyeloid progenitors derived from embryonic yolk sac[8], 4hydroxytamoxifen (4-OHT from Sigma) was administered i.p. to pregnant females on E9.5 at a dose of $75 \mu \mathrm{g} / \mathrm{g}$ (body weight). To improve the survival of embryos and reduce the risk of abortions, Progesterone (Sigma) was co-

868 injected at a dose of $37.5 \mu \mathrm{g} / \mathrm{g}$ (body weight)[91]. To label the $C \times 3 c r 1^{+}$thymic 869 macrophages in $C \times 3 c r 1^{C r e E R} \times R O S A 26^{L S L-G F P}$ neonates and adult mice,

870 Tamoxifen (TAM from Sigma) was injected i.p. at a dose of $2 \mathrm{mg} / \mathrm{mouse}$ to 871 lactating dams on postnatal day 3 and 4 (P3 and P4) or to adult mice for 2 872 consecutive days. Thymuses were harvested and analyzed 3 days or 6 873 weeks after the last injection by flow cytometry. 


\section{scRNA-Seq - sorting, library generation, and sequencing}

877 Biotechnological Applications of NCFB (NYCU, Taipei, Taiwan). Briefly, the thymuses of one female MAFIA and 2 male $C d 11 c^{Y F P}$ mice were harvested and enzymatically digested as described previously. Mononuclear cells were enriched by $57 \%$ Percoll PLUS discontinuous centrifugation, washed to

881 remove silica particles, and resuspended at $10^{6} / \mathrm{mL}$ in PBS with $0.04 \%$ BSA.

882 The cell suspensions were filtered through Falcon $35 \mu \mathrm{m}$ strainer (Corning) 883 and stained with viability dye (PI or DAPI) immediately before sorting. Cell sorting was performed on a FACS Melody sorter (BD Biosciences) running FACS Chorus (BD Biosciences) software in purity mode. $3 \times 10^{5}$ GFP or YFP positive cells under the live/singlet gating were collected into $5 \mathrm{ml}$ round

887 bottom tubes pre-coated with $0.04 \%$ BSA in PBS. Sorted cells were washed 888 and resuspended in $300 \mu \mathrm{L}$ PBS with $0.04 \%$ BSA and then filtered again into 1.5-mL DNA LoBind tubes (Eppendorf) through a $35 \mu \mathrm{m}$ strainer. The viability of the cells was evaluated by Countess II (Invitrogen) and Trypan Blue

891 (ThermoFisher), and samples with cell viability rates higher than $85 \%$ were 892 used for encapsulation and library preparation. Single-cell encapsulation and 893 library preparation were performed using Single Cell 3' v3/v3.1 Gene 894 Expression solution (10x Genomics). All the libraries were processed according to the manufacturer's instruction and sequenced on NovaSeq 6000

896 (Illumina) platform at the NHRI (Zhubei, Taiwan). Post-processing and quality 897 control were performed by the NYCU Genome Center using the CellRanger 898 package (v. 3.0.2, 10x Genomics). Reads were aligned to $\mathrm{mm} 10$ reference 899 assembly. Primary assessment with CellRanger reported 9,973 cell-barcodes 
with 11,385 median unique molecular identifiers (UMIs, transcripts) per cell

901 and 3,076 median genes per cell sequenced to $71.0 \%$ sequencing saturation

with 94,260 mean reads per cell for MAFIA mouse sample; 9,801 cell-

barcodes with 13,467 median UMls per cell and 3,211 median genes per cell

904 sequenced to $74.9 \%$ sequencing saturation with 119,820 mean reads per cell

905 for the first $C d 11 c^{Y F P}$ mouse sample; 12,938 cell-barcodes with 14,439

906 median UMls per cell and 3,199 median genes per cell sequenced to $71.4 \%$

907 sequencing saturation with 108,585 mean reads per cell for the second

908 Cd11 $c^{\text {YFP }}$ mouse sample.

$910 \quad$ Analysis of scRNA-Seq

$911 \quad$ Preprocessing

912 The Scanpy[92] pipeline was used to read the count matrix. Three batches

913 of samples (one from $\mathrm{GFP}^{+}$cells from MAFIA mouse and two from $\mathrm{YFP}^{+}$cells

914 from Cd11 ${ }^{\text {YFP }}$ mice) were preprocessed independently and integrated later.

915 Cells that expressed $<200$ genes and genes that were expressed in $<3$ cells

916 were filtered out. The percentage of mitochondrial genes was calculated -and

917 cells with $>10 \%$ mitochondrial genes were removed. Cells with $>7,000$ genes

918 or $<1,000$ genes were also removed. Read counts were normalized to library

919 size 10,000 and log-transformed with scanpy.pp.log1p function.

920 Datasets integration and batch effect correction

921 Read count matrices and spliced/unspliced matrices were merged first.

922 Principal Component Analysis was applied to reduce dimensions to 70.

923 BBKNN[93] was then used to remove batch effects with the

924 scanpy.external.pp.bbknn function with default parameters. 
UMAP[94] provided by scanpy was used to visualize data with default

927 parameters. K-nearest neighbor and Leiden clustering were applied

928 sequentially to cluster cells into groups. K-nearest neighbor graph

929 construction was done by scanpy.pp.neighbors with parameters

930 n_neighbors $=12$ and $n \_p c s=70$. Leiden clustering was then performed by

931 scanpy.tl.leiden with parameter resolution=0.15. To improve UMAP

932 visualization, scanpy.tl.paga was applied, and we trimmed unnecessary graph

933 edges by scanpy.tl.paga with threshold=0.018.

$934 \quad$ Marker genes and statistics

935 Wilcoxon rank-sum tests were applied to examine differentially expressed

936 genes. Clusters were selected from the result of Leiden clustering.

937 Differentially expressed genes of a cluster against other clusters were

938 identified by scanpy.tl.rank_genes_groups and scanpy.pl.rank_genes_groups.

939 P-values were collected for each cluster and transformed by negative $\log _{10}$ for

940 better visualization. The top 50 differentially expressed genes were visualized

941 in the figure.

\section{Immunofluorescent staining}

944 Dissected thymus lobes from C57BL/6 mice were cleaned of connective

945 tissue and fixed in $4 \%$ paraformaldehyde (Sigma) for $1 \mathrm{~h}$ at $4^{\circ} \mathrm{C}$, washed in

946 PBS, submerged in $10 \%$ sucrose, and then in $30 \%$ sucrose for $12 \mathrm{~h}$ each.

947 The tissue was then frozen in Tissue-Tek OCT compound (Sakura Fintek) for

948 cryostat sectioning. 10 or $20 \mu \mathrm{m}$ thick sections were prepared with CryoStar

949 NX50 (ThermoFisher) on Superfrost PLUS (ThermoScientific) microscope 
950 slides, dried overnight, and stored at $-80^{\circ} \mathrm{C}$ until used. Before staining, the

951 sections were fixed with acetone (Sigma) at $-20^{\circ} \mathrm{C}$ for $10 \mathrm{~min}$, air-dried, then

952 blocked with $5 \%$ goat serum $+5 \%$ donkey serum (both from Jackson

953 Immunoresearch) in PBS for $2 \mathrm{~h}$ and stained with primary antibodies: rat

954 monoclonal to MerTK (DS5MMER, eBioscience), rat monoclonal to TIM4

955 (RMT4-54, Bio-X-Cell) or rabbit polyclonal to Keratin 5 (BioLegend) overnight

956 at $4^{\circ} \mathrm{C}$ in a humidified chamber. After washing in PBS, the sections were

957 labeled with goat anti-rat-Alexa Fluor 647 (Invitrogen) or goat anti-rat Cy3

958 (Jackson Immunoresearch) and donkey anti-rabbit AF647 (Jackson

959 Immunoresearch) secondary antibodies for 2 hours at room temperature,

960 followed by 5 min staining with DAPI. TUNEL Assay was done with the Click-

961 iT Plus TUNEL Assay Alexa Fluor 647 kit (Invitrogen) according to the

962 manufacturer's recommendations. A positive (pre-incubation with DNase I for

$96330 \mathrm{~min}$ at room temperature) and negative (no TdT enzyme) controls were

964 always included. The sections were mounted with $0.1 \% \mathrm{n}$-propyl gallate

965 (Sigma) in glycerol (Sigma) and imaged with an AxioObserver 7 (Carl Zeiss)

966 wide-field microscope equipped with Plan Apochromat 20x NA=0.8 objective

967 (Zeiss) and AxioCam 702 mono camera (Zeiss) and controlled by Zen 2.3

968 Blue (Zeiss) software. Image analysis was performed with Imaris 8.0.2

969 (Bitplane).

971 The co-localization scoring for MerTK and TIM4 with TUNEL was done with

972 Imaris 8.2 (Bitplane). TUNEL ${ }^{+}$cells were detected with the Spots function,

973 while $\mathrm{MerTK}^{+}$and $\mathrm{TIM}^{+}$cells were detected with the Surface function. Spots

974 that co-localize with Surfaces were identified with the "Find Spots close to 
975 Surface" function of Imaris XT. The threshold for co-localization was set to 5

$976 \mu \mathrm{m}$. The results were manually curated so that Spots categorized as "not co-

977 localized" that were: 1) at the edge of the imaging field were excluded from

978 consideration; 2) with clear Surface signal around them were re-categorized

979 as "co-localized". The ratio of co-localized Spots to all Spots was calculated

980 and presented as the co-localization index.

\section{Thymus transplantation}

To obtain E15.5 embryos, ROSA26 GFP homozygous male and C57BL/6

984 female mice were mated in a cage overnight and separated on the next day.

985 Pregnant mice were sacrificed 15 days later, the viable embryos were

986 harvested, and the thymuses were isolated in ice-cold PBS. C57BL/6

987 recipients were anesthetized by i.p injection of Ketamine hydrochloride (120

$988 \mu \mathrm{g} / \mathrm{g}$, Toronto Research Chemicals) and Xylazine hydrochloride (12 $\mu \mathrm{g} / \mathrm{g}$,

989 Sigma). The fur on the left flank was removed, and the left kidney was

990 exposed by cutting the skin, muscle layer, and peritoneum. The kidney

991 capsule was nicked with a G23 needle, and the fetal thymus was pushed into

992 the pocket under the kidney capsule with a G23 needle equipped with a

993 plunger from a spinal needle. After the kidney was re-positioned back into the

994 peritoneal cavity, the peritoneum was sutured, and the skin was stapled with

995 metal clips. Rymadil (Carprofen, $5 \mu \mathrm{g} / \mathrm{g}$, Zoetis) was given subcutaneously to

996 ease the wound pain, and Trimerin (Sulfadiazine at $0.5 \mathrm{mg} / \mathrm{mL}+$ Trimethoprim

997 at $0.1 \mathrm{mg} / \mathrm{mL}$ ) were given in the drinking water for the first two weeks after the

998 surgery. The metal clips were removed from the skin after the first week, and 

the transplanted thymus and recipient's endogenous thymus were harvested and analyzed six weeks after the kidney transplantation.

\section{Statistical analysis}

Comparison between groups was made with Prism 6 (GraphPad Software). Comparisons between two groups were carried out with unpaired Student's ttest. When more than two groups were compared, a one-way ANOVA with Tukey correction was used. Differences were considered significant if $p<0.05$.

\section{Data availability}

The RNA Sequencing data of thymic macrophages and thymic dendritic cells is available at NCBI Gene Expression Omnibus (GEO) as part of

GSE122108 and at www.immgen.org. The single cell RNA sequencing data is deposited at NCBI GEO under accession number GSE185460. The source data underlying Fig. 1g-h, Fig. 3c, f, i, Fig. 4b. f, h, Fig. 6b, d, Fig. 7b, d, e, Fig. S1h, Fig. S3c, and Fig. S4b, d, e, g are provided in the Source Data files. All other data supporting the findings of this study are available within the article and its figures and tables.

\section{Abbreviations:}

cDC - classical dendritic cell

$$
\text { DC - dendritic cell }
$$

$$
\text { EdU - 5-Ethynyl-2'-deoxyuridine }
$$

$$
\text { GO - gene ontology }
$$

HSC - hematopoietic stem cell 
1024 IFN-I - type I Interferon

1025 IMMGEN - Immunological Genome Consortium

1026 scRNA-Seq - single-cell RNA sequencing

$1027 \quad$ TF - transcription factor

1028 ThyMacs - thymic macrophages

1029 TRA - tissue-restricted antigen

$1030 \quad$ YS - yolk sac

\section{References:}

1032

1033

1034

1035

1036

1037

1038

1039

1040

1041

1042

1043

1044

1045

1046

1047

1048

1049

1050

1051

1052

1053

1054

1055

1056

1057

1. Wynn TA, Chawla A, Pollard JW. Macrophage biology in development, homeostasis and disease. Nature. Nature Publishing Group; 2013;496: 445-455. doi:10.1038/nature12034

2. Baratin M, Simon L, Jorquera A, Ghigo C, Dembele D, Nowak J, et al. T Cell Zone Resident Macrophages Silently Dispose of Apoptotic Cells in the Lymph Node. Immunity. Elsevier Inc; 2017;47: 349-362.e5. doi:10.1016/j.immuni.2017.07.019

3. A-Gonzalez N, Castrillo A. Origin and Specialization of Splenic Macrophages. Cellular Immunology. 2018;330: 1-27. doi:10.1016/j.cellimm.2018.05.005

4. Bellomo A, Mondor I, Spinelli L, Lagueyrie M, Stewart BJ, Brouilly N, et al. Reticular Fibroblasts Expressing the Transcription Factor WT1 Define a Stromal Niche that Maintains and Replenishes Splenic Red Pulp Macrophages. Immunity. Elsevier Inc; 2020;53: 127-142.e7. doi:10.1016/j.immuni.2020.06.008

5. van Furth $\mathrm{R}, \mathrm{Cohn} Z \mathrm{ZA}$. The origin and kinetics of mononuclear phagocytes. J Exp Med. Rockefeller University Press; 1968;128: 415435. doi:10.1084/jem.128.3.415

6. Ginhoux F, Guilliams M. Tissue-Resident Macrophage Ontogeny and Homeostasis. Immunity. 2016;44: 439-449.

doi:10.1016/j.immuni.2016.02.024

7. Perdiguero EG, Klapproth K, Schulz C, Busch K, Azzoni E, Crozet L, et al. Tissue-resident macrophages originate from yolk-sac-derived erythro-myeloid progenitors. Nat Immunol. Nature Publishing Group; 2015;518: 547-551. doi:10.1038/nature13989

8. Mass E, Ballesteros I, Farlik M, Halbritter F, Günther P, Crozet L, et al. 
1058

1059

1060

1061

1062

1063

1064

1065

1066

1067

1068

1069

1070

1071

1072

1073

1074

1075

1076

1077

1078

1079

1080

1081

1082

1083

1084

1085

1086

1087

1088

1089

1090

1091

1092

1093

1094

1095

1096

1097

1098

Specification of tissue-resident macrophages during organogenesis.

Science. American Association for the Advancement of Science; 2016;353: aaf4238-aaf4238. doi:10.1126/science.aaf4238

9. Ginhoux F, Greter M, Leboeuf M, Nandi S, See P, Gokhan S, et al. Fate mapping analysis reveals that adult microglia derive from primitive macrophages. Science. 2010;330: 841-845.

doi:10.1126/science.1194637

10. Hoeffel G, Wang Y, Greter M, See P, Teo P, Malleret B, et al. Adult Langerhans cells derive predominantly from embryonic fetal liver monocytes with a minor contribution of yolk sac-derived macrophages. J Exp Med. 2012;209: 1167-1181. doi:10.1084/jem.20120340

11. Hoeffel G, Chen J, Lavin Y, Low D, Almeida FF, See P, et al. C-Myb(+) erythro-myeloid progenitor-derived fetal monocytes give rise to adult tissue-resident macrophages. Immunity. 2015;42: 665-678.

doi:10.1016/j.immuni.2015.03.011

12. Goldmann T, Wieghofer P, Jordão MJC, Prutek F, Hagemeyer N, Frenzel K, et al. Origin, fate and dynamics of macrophages at central nervous system interfaces. Nat Immunol. 2016;17: 797-805. doi:10.1038/ni.3423

13. Hashimoto D, Chow A, Noizat C, Teo P, Beasley MB, Leboeuf M, et al. Tissue-Resident Macrophages Self-Maintain Locally throughout Adult Life with Minimal Contribution from Circulating Monocytes. Immunity. Elsevier Inc; 2013;38: 792-804. doi:10.1016/j.immuni.2013.04.004

14. Epelman S, Lavine KJ, Beaudin AE, Sojka DK, Carrero JA, Calderon B, et al. Embryonic and Adult-Derived Resident Cardiac Macrophages Are Maintained through Distinct Mechanisms at Steady State and during Inflammation. Immunity. Elsevier Inc; 2014;40: 91-104. doi:10.1016/j.immuni.2013.11.019

15. Sheng J, Ruedl C, Karjalainen K. Most Tissue-Resident Macrophages Except Microglia Are Derived from Fetal Hematopoietic Stem Cells. Immunity. Elsevier Inc; 2015;43: 382-393.

doi:10.1016/j.immuni.2015.07.016

16. Liu Z, Gu Y, Chakarov S, Bleriot C, Kwok I, Chen X, et al. Fate Mapping via Ms4a3-Expression History Traces Monocyte-Derived Cells. Cell. Elsevier; 2019;178: 1509-1525.e19. doi:10.1016/j.cell.2019.08.009

17. Ensan S, Li A, Besla R, Degousee N, Cosme J, Roufaiel M, et al. Selfrenewing resident arterial macrophages arise from embryonic CX3CR1+ precursors and circulating monocytes immediately after birth. Nat Immunol. Nature Publishing Group; 2015;17: 159-168. doi:10.1038/ni.3343

18. Mondor I, Baratin M, Lagueyrie M, Saro L, Henri S, Gentek R, et al. 

Lymphatic Endothelial Cells Are Essential Components of the Subcapsular Sinus Macrophage Niche. Immunity. Elsevier Inc; 2019;50: 1453-1466.e4. doi:10.1016/j.immuni.2019.04.002

19. Molawi K, Wolf Y, Kandalla PK, Favret J, Hagemeyer N, Frenzel K, et al. Progressive replacement of embryo-derived cardiac macrophages with age. J Exp Med. 2014;211: 2151-2158. doi:10.1084/jem.20140639

20. Heidt T, Courties G, Dutta P, Sager HB, Sebas M, Iwamoto $Y$, et al. Differential Contribution of Monocytes to Heart Macrophages in SteadyState and After Myocardial Infarction. Circulation Research. 2014;115: 284-295. doi:10.1161/CIRCRESAHA.115.303567

21. Yahara $Y$, Barrientos $T$, Tang $Y J$, Puviindran $V$, Nadesan $P$, Zhang $H$, et al. Erythromyeloid progenitors give rise to a population of osteoclasts that contribute to bone homeostasis and repair. Nat Cell Biol. Springer US; 2020;22: 1-28. doi:10.1038/s41556-019-0437-8

22. Jacome-Galarza CE, Percin GI, Muller JT, Mass E, Lazarov T, Eitler J, et al. Developmental origin, functional maintenance and genetic rescue of osteoclasts. Nature. Nature Publishing Group; 2019;568: 541-545. doi:10.1038/s41586-019-1105-7

23. Calderon B, Carrero JA, Ferris ST, Sojka DK, Moore L, Epelman S, et al. The pancreas anatomy conditions the origin and properties of resident macrophages. J Exp Med. 2015;212: 1497-1512. doi:10.1084/jem.20150496

24. Tamoutounour S, Guilliams M, Sanchis FM, Liu H, Terhorst D, Malosse $\mathrm{C}$, et al. Origins and Functional Specialization of Macrophages and of Conventional and Monocyte-Derived Dendritic Cells in Mouse Skin. Immunity. Elsevier Inc; 2013;39: 925-938. doi:10.1016/j.immuni.2013.10.004

25. Bain CC, Bravo-Blas A, Scott CL, Gomez Perdiguero E, Geissmann F, Henri S, et al. Constant replenishment from circulating monocytes maintains the macrophage pool in the intestine of adult mice. Nat Immunol. 2014;15: 929-937. doi:10.1038/ni.2967

26. Sierro F, Evrard M, Rizzetto S, Melino M, Mitchell AJ, Florido M, et al. A Liver Capsular Network of Monocyte-Derived Macrophages Restricts Hepatic Dissemination of Intraperitoneal Bacteria by Neutrophil Recruitment. Immunity. Elsevier Inc; 2017;47: 374-388.e6. doi:10.1016/j.immuni.2017.07.018

27. Mossadegh-Keller N, Gentek R, Gimenez G, Bigot S, Mailfert S, Sieweke $\mathrm{MH}$. Developmental origin and maintenance of distinct testicular macrophage populations. J Exp Med. 2017;214: 2829-2841. doi:10.1084/jem.20170829

28. Lokka E, Lintukorpi L, Cisneros-Montalvo S, Mäkelä J-A, Tyystjärvi S, 
Ojasalo V, et al. Generation, localization and functions of macrophages during the development of testis. Nature Communications. Springer US; 2020;11: 1-16. doi:10.1038/s41467-020-18206-0

29. Wang M, Yang Y, Cansever D, Wang Y, Kantores C, Messiaen S, et al. Two populations of self-maintaining monocyte-independent macrophages exist in adult epididymis and testis. PNAS. 2020;118: e2013686117. doi:10.1073/pnas.2013686117/-/DCSupplemental

30. Bain CC, Hawley CA, Garner H, Scott CL, Schridde A, Steers NJ, et al. Long-lived self-renewing bone marrow-derived macrophages displace embryo-derived cells to inhabit adult serous cavities. Nature Communications. Nature Publishing Group; 2016;7: 1-14. doi:10.1038/ncomms11852

31. Surh CD, Sprent J. T-cell apoptosis detected in situ during positive and negative selection in the thymus. Nature. 1994;372: 100-103. doi:10.1038/372100a0

32. Esashi E, Sekiguchi T, Ito H, Koyasu S, Miyajima A. Cutting Edge: A possible role for $\mathrm{CD} 4+$ thymic macrophages as professional scavengers of apoptotic thymocytes. Journal of Immunology (Baltimore, Md : 1950). American Association of Immunologists; 2003;171: 2773-2777. doi:10.4049/jimmunol.171.6.2773

33. Liu L-T, Lang Z-F, Li Y, Zhu Y-J, Zhang J-T, Guo S-F, et al. Composition and characteristics of distinct macrophage subpopulations in the mouse thymus. Molecular Medicine Reports. 2013;7: 1850-1854. doi:10.3892/mmr.2013.1448

34. Guerri L, Peguillet I, Geraldo Y, Nabti S, Premel V, Lantz O. Analysis of APC types involved in CD4 tolerance and regulatory T cell generation using reaggregated thymic organ cultures. J Immunol. American Association of Immunologists; 2013;190: 2102-2110. doi:10.4049/jimmunol.1202883

35. Lopes N, Charaix J, Cédile O, Sergé A, Irla M. Lymphotoxin alpha finetunes $\mathrm{T}$ cell clonal deletion by regulating thymic entry of antigenpresenting cells. Nature Communications. Springer US; 2018;9: 1-16. doi:10.1038/s41467-018-03619-9

36. Kim H-J, Alonzo ES, Dorothee G, Pollard JW, Sant'Angelo DB. Selective depletion of eosinophils or neutrophils in mice impacts the efficiency of apoptotic cell clearance in the thymus. Kim H-J, Alonzo ES, Dorothee G, Pollard JW, Sant'Angelo DB, editors. PLoS ONE. 2010;5: e11439. doi:10.1371/journal.pone.0011439.g010

37. Tacke R, Hilgendorf I, Garner H, Waterborg C, Park K, Nowyhed H, et al. The transcription factor NR4A1 is essential for the development of a novel macrophage subset in the thymus. Sci Rep. 2015;5: 10055. doi:10.1038/srep10055 
1182

1183

1184

1185

1186

1187

1188

1189

1190

1191

1192

1193

1194

1195

1196

1197

1198

1199

1200

1201

1202

1203

1204

1205

1206

1207

1208

1209

1210

1211

1212

1213

1214

1215

1216

1217

1218

1219

1220

1221

1222

38. Gautier EL, Shay T, Miller J, Greter M, Jakubzick C, Ivanov S, et al. Gene-expression profiles and transcriptional regulatory pathways that underlie the identity and diversity of mouse tissue macrophages. Nat Immunol. 2012;13: 1118-1128. doi:10.1038/ni.2419

39. Ingersoll MA, Spanbroek R, Lottaz C, Gautier EL, Frankenberger M, Hoffmann $\mathrm{R}$, et al. Comparison of gene expression profiles between human and mouse monocyte subsets. Blood. 2010;115: e10-e19. doi:10.1182/blood-2009-07-235028

40. Wang H, Breed ER, Lee YJ, Qian LJ, Jameson SC, Hogquist KA. Myeloid cells activate iNKT cells to produce IL-4 in the thymic medulla. Proc Natl Acad Sci. National Academy of Sciences; 2019;116: 2226222268. doi:10.1073/pnas.1910412116

41. Chan CT, Fenn AM, Harder NK, Mindur JE, McAlpine CS, Patel J, et al. Liver $\mathrm{X}$ receptors are required for thymic resilience and T cell output. J Exp Med. 2020;217: 245-23. doi:10.1084/jem.20200318

42. Wada H, Masuda K, Satoh R, Kakugawa K, Ikawa T, Katsura Y, et al. Adult T-cell progenitors retain myeloid potential. Nature. Nature Publishing Group; 2008;452: 768-772. doi:10.1038/nature06839

43. Bell JJ, Bhandoola A. The earliest thymic progenitors for T cells possess myeloid lineage potential. Nature. 2008;452: 764-767. doi:10.1038/nature06840

44. Schlenner SM, Madan V, Busch K, Tietz A, LAufle C, Costa C, et al. Fate Mapping Reveals Separate Origins of T Cells and Myeloid Lineages in the Thymus. Immunity. Elsevier Ltd; 2010;32: 426-436. doi:10.1016/j.immuni.2010.03.005

45. Witmer-Pack MD, Hughes DA, Schuler G, Lawson L, McWilliam A, Inaba K, et al. Identification of macrophages and dendritic cells in the osteopetrotic (op/op) mouse. J Cell Sci. 1993;104 ( Pt 4): 1021-1029.

46. Sasmono RT, Oceandy D, Pollard JW, Tong W, Pavli P, Wainwright BJ, et al. A macrophage colony-stimulating factor receptor-green fluorescent protein transgene is expressed throughout the mononuclear phagocyte system of the mouse. Blood. American Society of Hematology; 2003;101: 1155-1163. doi:10.1182/blood-2002-02-0569

47. Hume DA. Applications of myeloid-specific promoters in transgenic mice support in vivo imaging and functional genomics but do not support the concept of distinct macrophage and dendritic cell lineages or roles in immunity. Journal of Leukocyte Biology. 2011;89: 525-538. doi:10.1189/jlb.0810472

48. Taniuchi I, Osato M, Egawa T, Sunshine MJ, Bae SC, Komori T, et al. Differential requirements for Runx proteins in CD4 repression and epigenetic silencing during T lymphocyte development. Cell. 2002;111: 
1224

1225

1226

1227

1228

1229

1230

1231

1232

1233

1234

1235

1236

1237

1238

1239

1240

1241

1242

1243

1244

1245

1246

1247

1248

1249

1250

1251

1252

1253

1254

1255

1256

1257

1258

1259

1260

1261

1262

1263

49. Ebihara T, Song C, Ryu SH, Plougastel-Douglas B, Yang L, Levanon D, et al. Runx3 specifies lineage commitment of innate lymphoid cells. Nat Immunol. Nature Publishing Group; 2015;16: 1124-1133. doi:10.1038/ni.3272

50. Fainaru O, Woolf E, Lotem J, Yarmus M, Brenner O, Goldenberg D, et al. Runx3 regulates mouse TGF-beta-mediated dendritic cell function and its absence results in airway inflammation. EMBO J. John Wiley \& Sons, Ltd; 2004;23: 969-979. doi:10.1038/sj.emboj.7600085

51. Kohyama M, Ise W, Edelson BT, Wilker PR, Hildner K, Mejia C, et al. Role for Spi-C in the development of red pulp macrophages and splenic iron homeostasis. Nat Immunol. Nature Publishing Group; 2008;457: 318-321. doi:10.1038/nature07472

52. Haldar M, Kohyama M, So AY-L, KC W, Wu X, Briseño CG, et al. Heme-Mediated SPI-C Induction Promotes Monocyte Differentiation into Iron-Recycling Macrophages. Cell. Elsevier Inc; 2014;156: 1223-1234. doi:10.1016/j.cell.2014.01.069

53. Yona S, Kim K-W, Wolf Y, Mildner A, Varol D, Breker M, et al. Fate Mapping Reveals Origins and Dynamics of Monocytes and Tissue Macrophages under Homeostasis. Immunity. Elsevier; 2013;38: 79-91. doi:10.1016/j.immuni.2012.12.001

54. McCaughtry TM, Wilken MS, Hogquist KA. Thymic emigration revisited. J Exp Med. 2007;204: 2513-2520. doi:10.1084/jem.20070601

55. Lienenklaus S, Cornitescu M, Ziętara N, Łyszkiewicz M, Gekara N, Jabłónska J, et al. Novel reporter mouse reveals constitutive and inflammatory expression of IFN-beta in vivo. J Immunol. American Association of Immunologists; 2009;183: 3229-3236. doi:10.4049/jimmunol.0804277

56. Otero DC, Baker DP, David M. IRF7-dependent IFN- $\beta$ production in response to RANKL promotes medullary thymic epithelial cell development. J Immunol. American Association of Immunologists; 2013;190: 3289-3298. doi:10.4049/jimmunol.1203086

57. Breed ER, Lee ST, Hogquist KA. Directing T cell fate: How thymic antigen presenting cells coordinate thymocyte selection. Seminars in Cell and Developmental Biology. Elsevier Ltd; 2017;: 1-9. doi:10.1016/j.semcdb.2017.07.045

58. Kurd NS, Lutes LK, Yoon J, Chan SW, Dzhagalov IL, Hoover AR, et al. A role for phagocytosis in inducing cell death during thymocyte negative selection. eLife. 2019;8: 1879-18. doi:10.7554/eLife.48097

59. Morioka S, Perry JSA, Raymond MH, Medina CB, Zhu Y, Zhao L, et al. Efferocytosis induces a novel SLC program to promote glucose uptake 
and lactate release. Nature. Springer US; 2018;563: 1-21.

doi:10.1038/s41586-018-0735-5

1266

1267

1268

1269

1270

1271

1272

1273

1274

1275

1276

1277

1278

1279

1280

1281

1282

1283

1284

1285

1286

1287

1288

1289

1290

1291

1292

1293

1294

1295

1296

1297

1298

1299

1300

1301

1302

1303

1304

60. Bleriot C, Chakarov S, Ginhoux F. Determinants of Resident Tissue Macrophage Identity and Function. Immunity. Elsevier Inc; 2020;52: 957-970. doi:10.1016/j.immuni.2020.05.014

61. Stritesky GL, Xing Y, Erickson JR, Kalekar LA, Wang X, Mueller DL, et al. Murine thymic selection quantified using a unique method to capture deleted T cells. Proc Natl Acad Sci. 2013;110: 4679-4684. doi:10.1073/pnas.1217532110

62. Baba T, Nakamoto Y, Mukaida N. Crucial Contribution of Thymic Sirp + Conventional Dendritic Cells to Central Tolerance against Blood-Borne Antigens in a CCR2-Dependent Manner. J Immunol. 2009;183: 30533063. doi:10.4049/jimmunol.0900438

63. Gallegos AM, Bevan MJ. Central Tolerance to Tissue-specific Antigens Mediated by Direct and Indirect Antigen Presentation. J Exp Med. 2004;200: 1039-1049. doi:10.1084/jem.20041457

64. Koble C, Kyewski B. The thymic medulla: a unique microenvironment for intercellular self-antigen transfer. J Exp Med. 2009;206: 1505-1513. doi:10.1084/jem.20082449

65. Vobořil M, Brabec T, Dobeš J, Šplíchalová I, Březina J, Čepková A, et al. Toll-like receptor signaling in thymic epithelium controls monocytederived dendritic cell recruitment and Treg generation. Nature Communications. Springer US; 2020;11: 1-16. doi:10.1038/s41467020-16081-3

66. Dixit VD. Impact of immune-metabolic interactions on age-related thymic demise and T cell senescence. Semin Immunol. Elsevier Ltd; 2012;24: 321-330. doi:10.1016/j.smim.2012.04.002

67. Rivera-Gonzalez GC, Shook BA, Andrae J, Holtrup B, Bollag K, Betsholtz C, et al. Skin Adipocyte Stem Cell Self-Renewal Is Regulated by a PDGFA/AKT-Signaling Axis. Stem Cell. Elsevier Inc; 2016;19: 738-751. doi:10.1016/j.stem.2016.09.002

68. Olson LE, Soriano P. Increased PDGFR\&alpha; Activation Disrupts Connective Tissue Development and Drives Systemic Fibrosis.

Developmental Cell. Elsevier Ltd; 2009;16: 303-313. doi:10.1016/j.devcel.2008.12.003

69. Satpathy AT, KC W, Albring JC, Edelson BT, Kretzer NM, Bhattacharya $\mathrm{D}$, et al. Zbtb46expression distinguishes classical dendritic cells and their committed progenitors from other immune lineages. J Exp Med. 2012;209: 1135-1152. doi:10.1084/jem.20120030

70. Guilliams M, Mildner A, Yona S. Developmental and Functional Heterogeneity of Monocytes. Immunity. Elsevier Inc; 2018;49: 595-613. 
1306

71. Tabula Muris Consortium. Single-cell transcriptomics of 20 mouse organs creates a Tabula Muris. Nature. Nature Publishing Group; 2018;562: 367-372. doi:10.1038/s41586-018-0590-4

72. Han X, Wang R, Zhou Y, Fei L, Sun H, Lai S, et al. Mapping the Mouse Cell Atlas by Microwell-Seq. Cell. Elsevier Inc; 2018;172: 10911097.e17. doi:10.1016/j.cell.2018.02.001

73. Tabula Muris Consortium. A single-cell transcriptomic atlas characterizes ageing tissues in the mouse. Nature. Nature Publishing Group; 2020;583: 590-595. doi:10.1038/s41586-020-2496-1

74. Kernfeld EM, Genga RMJ, Neherin K, Magaletta ME, Xu P, Maehr R. A Single-Cell Transcriptomic Atlas of Thymus Organogenesis Resolves Cell Types and Developmental Maturation. Immunity. Elsevier Inc; 2018;: 1-33. doi:10.1016/j.immuni.2018.04.015

75. Park J-E, Botting RA, Domínguez Conde C, Popescu D-M, Lavaert M, Kunz DJ, et al. A cell atlas of human thymic development defines $T$ cell repertoire formation. Science. American Association for the Advancement of Science; 2020;367: eaay3224-12. doi:10.1126/science.aay3224

76. Bornstein C, Nevo S, Giladi A, Kadouri N, Pouzolles M, Gerbe F, et al. Single-cell mapping of the thymic stroma identifies IL-25-producing tuft epithelial cells. Nature. Springer US; 2018;559: 1-25. doi:10.1038/s41586-018-0346-1

77. Bautista JL, Cramer NT, Miller CN, Chavez J, Berrios DI, Byrnes LE, et al. Single-cell transcriptional profiling of human thymic stroma uncovers novel cellular heterogeneity in the thymic medulla. Nature Communications. Nature Publishing Group; 2021;12: 1096-15. doi:10.1038/s41467-021-21346-6

78. Burnett SH, Kershen EJ, Zhang J, Zeng L, Straley SC, Kaplan AM, et al. Conditional macrophage ablation in transgenic mice expressing a Fas-based suicide gene. Journal of Leukocyte Biology. Society for Leukocyte Biology; 2004;75: 612-623. doi:10.1189/jlb.0903442

79. Jung S, Aliberti J, Graemmel P, Sunshine MJ, Kreutzberg GW, Sher A, et al. Analysis of fractalkine receptor CX(3)CR1 function by targeted deletion and green fluorescent protein reporter gene insertion. Mol Cell Biol. 2000;20: 4106-4114.

80. Lindquist RL, Shakhar G, Dudziak D, Wardemann H, Eisenreich T, Dustin ML, et al. Visualizing dendritic cell networks in vivo. Nat Immunol. 2004;5: 1243-1250. doi:10.1038/ni1139

81. Faust N, Varas F, Kelly LM, Heck S, Graf T. Insertion of enhanced green fluorescent protein into the lysozyme gene creates mice with 
green fluorescent granulocytes and macrophages. Blood. 2000;96: 719-726.

82. Roesch K, Jadhav AP, Trimarchi JM, Stadler MB, Roska B, Sun BB, et al. The transcriptome of retinal Müller glial cells. J Comp Neurol. 2008;509: 225-238. doi:10.1002/cne.21730

83. Madisen L, Zwingman TA, Sunkin SM, Oh SW, Zariwala HA, Gu H, et al. A robust and high-throughput Cre reporting and characterization system for the whole mouse brain. Nature Neuroscience. Nature Publishing Group; 2009;13: 133-140. doi:10.1038/nn.2467

84. Picelli S, Faridani OR, rklund ASKBO, Winberg GOS, Sagasser S, Sandberg R. Full-length RNA-seq from single cells using Smart-seq2. Nature Protocols. Nature Publishing Group; 2014;9: 171-181. doi:10.1038/nprot.2014.006

85. Picelli S, Björklund ÅK, Faridani OR, Sagasser S, Winberg G, Sandberg R. Smart-seq2 for sensitive full-length transcriptome profiling in single cells. Nat Meth. 2013;10: 1096-1098. doi:10.1038/nmeth.2639

86. Love MI, Huber W, Anders S. Moderated estimation of fold change and dispersion for RNA-seq data with DESeq2. Genome Biol. BioMed Central; 2014;15: 31-21. doi:10.1186/s13059-014-0550-8

87. Schmeier S, Alam T, Essack M, Bajic VB. TcoF-DB v2: update of the database of human and mouse transcription co-factors and transcription factor interactions. Nucleic Acids Res. 2017;45: D145-D150. doi:10.1093/nar/gkw1007

88. Reich M, Liefeld T, Gould J, Lerner J, Tamayo P, Mesirov JP. GenePattern 2.0. Nat Genet. Nature Publishing Group; 2006;38: 500501. doi:10.1038/ng0506-500

89. Yu G, Wang L-G, Han Y, He Q-Y. clusterProfiler: an R Package for Comparing Biological Themes Among Gene Clusters. OMICS: A Journal of Integrative Biology. Mary Ann Liebert, Inc., publishers; 2012;16: 284-287. doi:10.1089/omi.2011.0118

90. Heyne GW, Plisch EH, Melberg CG, Sandgren EP, Peter JA, Lipinski RJ. A Simple and Reliable Method for Early Pregnancy Detection in Inbred Mice. J Am Assoc Lab Anim Sci. 2015;54: 368-371.

91. Iturri L, Saenz Coronilla J, Lallemand Y, Gomez Perdiguero E. Identification Of Erythromyeloid Progenitors And Their Progeny In The Mouse Embryo By Flow Cytometry. JoVE. 2017;: 1-10. doi:10.3791/55305

92. Wolf FA, Angerer P, Theis FJ. SCANPY: large-scale single-cell gene expression data analysis. Genome Biol. BioMed Central; 2018;19: 155. doi:10.1186/s13059-017-1382-0 
1411 to IL Dzhagalov), and the Cancer Progression Research Center (NYCU). The

93. Polański K, Young MD, Miao Z, Meyer KB, Teichmann SA, Park J-E. BBKNN: fast batch alignment of single cell transcriptomes. Bioinformatics. 2019;36: 964-965. doi:10.1093/bioinformatics/btz625\#supplementary-data

94. Mclnnes L, Healy J, Melville J. UMAP: Uniform Manifold Approximation and Projection for Dimension Reduction. ArXiv e-prints. 2018. doi:1802.03426v3

\section{Acknowledgments}

We are grateful to the IMMGEN consortium for performing the RNA sequencing of our samples and providing access to its database. We thank the following core facilities at National Yang-Ming Chiao-Tung University: Instrumentation Resource Center for providing access to its sorting and imaging facility and the Animal Facility for mouse husbandry. We are very grateful to Wan-Chun Chang, Bing-Xiu Guo, Chien-Yi Tung, and Kate Hua from the Genomics Center for Clinical and Biotechnological Applications of NCFB (NYCU, Taipei) for help with scRNA-Seq. Special thanks go to Dr. Fang Liao, Academia Sinica, Taipei, for help with cell sorting and for providing the 24G2 hybridoma, and Chang-Feng Chu for technical assistance. We are very grateful to Ms. Su-Hua Jiang at Veterans General Hospital, Taipei, for help with using the irradiator. Some figures were made with BioRender.

This work was supported by the Ministry of Science and Technology (grants \# 106-2320-B-010 -026 -MY3, and 107-2320-B-010 -016-MY3 to IL Dzhagalov and grants \# 107-2320-B-010-020, and 108-2628-B-010-005 to CL Hsu), the Yen-Tjing Ling Medical Foundation (grants \# Cl-107-6 and Cl-108-5

1412 authors declare no competing financial interests. 


\section{Author contributions}

$1414 \quad$ TA Zhou designed experiments, performed research, analyzed and

1415 interpreted data, and wrote the manuscript; HP Hsu and CY Lin performed

1416 research, analyzed and interpreted data; YH Tu and HC Huang analyzed the

1417 scRNA-Seq data; NJ Chen, JW Tsai, EA Robey, and CL Hsu provided

1418 expertise, shared critical reagents, and edited the manuscript; IL Dzhagalov

1419 conceptualized the studies, designed experiments, performed research,

1420 analyzed, interpreted the data, and wrote the manuscript.

\section{Supplementary figures and tables}

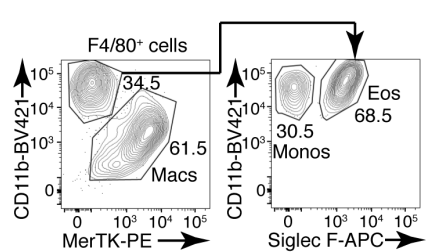

C

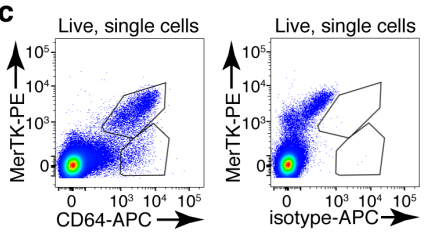

d

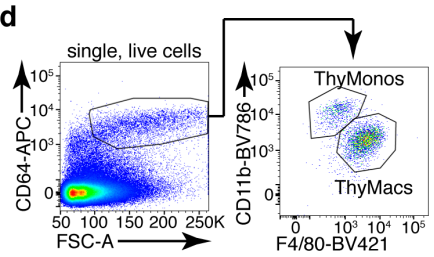

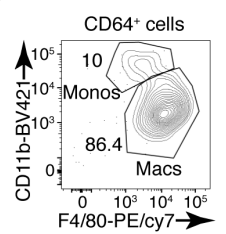
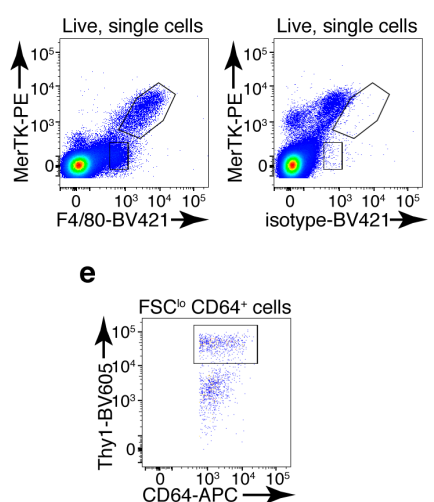

$\mathbf{f}$

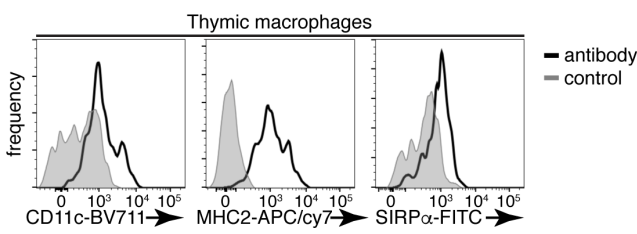

g

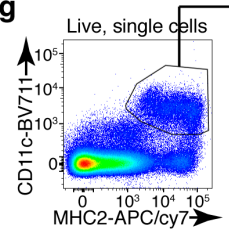

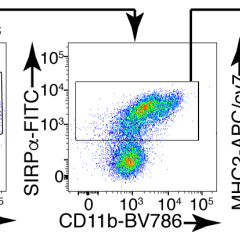

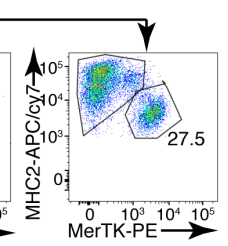

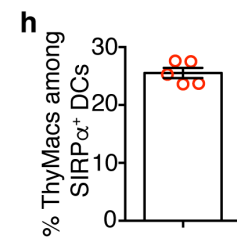

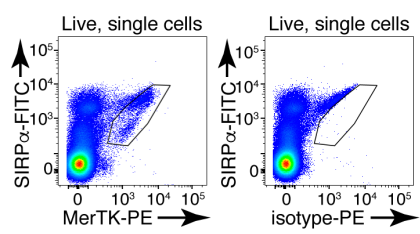


Figure 1 - Figure supplement 1: Phenotype of thymic macrophages

1425 and other myeloid cells positive for F4/80 and CD64. a Example flow

1426 cytometry plots breaking down $\mathrm{F} 4 / 80^{+}$cells in the thymus into macrophages

1427 (MerTK $\left.{ }^{+}\right)$, eosinophils $\left(\mathrm{CD}_{11 \mathrm{~b}^{+} \text {MerTK}}{ }^{-S i g l e c} \mathrm{~F}^{+}\right)$and monocytes

1428 (CD11 b+MerTK-Siglec $\left.\mathrm{F}^{-}\right)$. b An example flow cytometry plot dividing CD64+ 1429 cells into macrophages $\left(F 4 / 80^{+} \mathrm{CD} 11 \mathrm{~b}^{\mathrm{lo}}\right)$ and monocytes $\left(\mathrm{CD} 11 \mathrm{~b}^{+} \mathrm{F} 4 / 80^{\mathrm{lo}}\right)$. c

1430 Representative flow cytometry staining of enzymatically digested thymus

1431 single-cell suspension for CD64, MerTK, and F4/80 and respective isotype

1432 controls. d Gating strategy routinely used to identify thymic macrophages as

$1433 \mathrm{CD} 64^{+} \mathrm{F} 4 / 80^{+} \mathrm{CD} 11 \mathrm{~b}^{\text {lo }} \mathrm{FSC} \mathrm{C}^{\text {hi }}$ cells. e Example flow cytometry plots showing that $1434 \mathrm{CD} 4^{+} \mathrm{FSC}^{\text {lo }}$ cells include Thy $1^{+}$cells (most likely thymocytes). $\mathrm{f}$ Expression of

$1435 \mathrm{CD} 11 \mathrm{c}, \mathrm{MHC2}$, and SIRP $\alpha$ on ThyMacs with respective controls. g Example

1436 flow cytometry plots showing that gating on $\mathrm{CD} 11 \mathrm{c}^{+} \mathrm{MHC}^{+}$thymus cells, in 1437 addition to DCs, also includes macrophages, especially among SIRP $\alpha^{+}$cells.

1438 h Frequency of $\mathrm{MerTK}^{+}$cells among $\mathrm{CD} 11 \mathrm{c}^{+} \mathrm{MHC}^{+} \mathrm{SIRP} \alpha^{+}$cells. The data 1439 are mean \pm SEM from 5 individual mice. Each dot is an individual mouse. The 1440 flow cytometry plots are representative of $\geq 5$ individual experiments. 
a

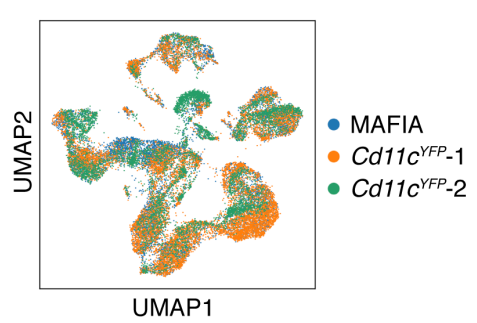

b
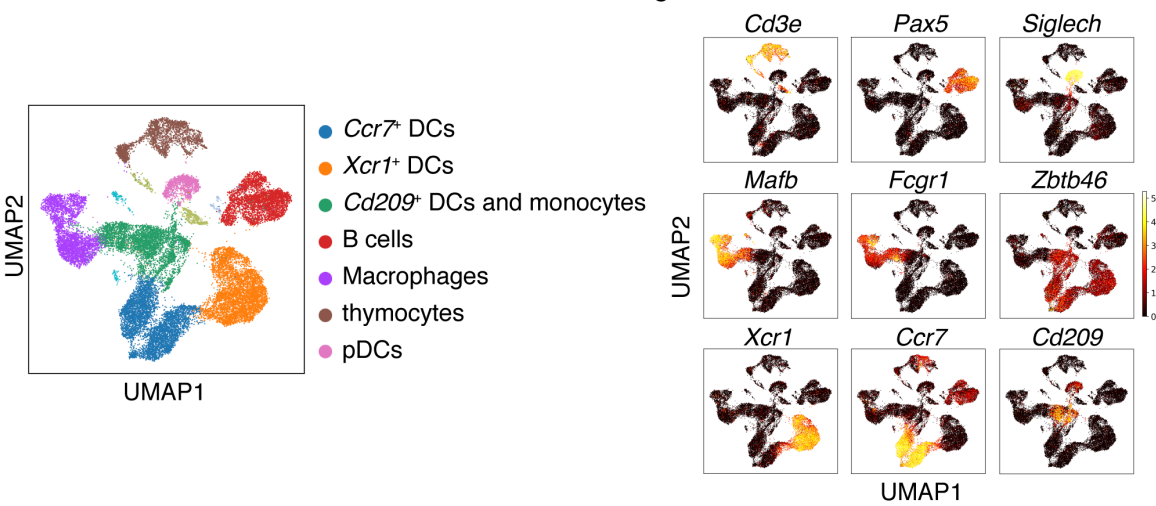

1442 Figure 1 - Figure Supplement 2: UMAP clustering of single-cell RNA-

1443 Sequencing (scRNA-Seq) data. a Clustering of the scRNA-Seq data shows

1444 that the cells from the three samples (one from GFP ${ }^{+}$cells in MAFIA mice and

1445 two from $\mathrm{YFP}^{+}$cells in $\mathrm{Cd11} \mathrm{c}^{\mathrm{YFP}}$ mice) overlap considerably. $\mathbf{b}$ Identification of

1446 the clusters from the scRNA-Seq data based on lineage-specific markers. c

1447 Expression of lineage-specific markers in different clusters.

1448 


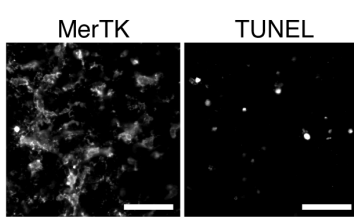

b

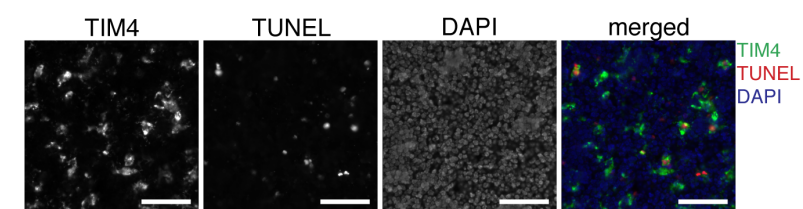

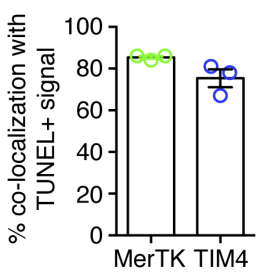

Figure 1 - Figure Supplement 3: Most TUNEL ${ }^{+}$apoptotic cells co-

1450 localize with thymic macrophages. a Example images showing co-

1451 localization of TUNEL ${ }^{+}$apoptotic cells and MerTK ${ }^{+}$ThyMacs. b Example

1452 images showing co-localization of TUNEL ${ }^{+}$apoptotic cells and TIM4 ${ }^{+}$

1453 ThyMacs. Scale bars in $\mathbf{a}$ and $\mathbf{b}$ are $50 \mu \mathrm{m}$. $\mathbf{c}$ Frequencies of the co-

1454 localization of TUNEL ${ }^{+}$signal with $\mathrm{MerTK}^{+}$and $\mathrm{TIM}^{+}$cells. All

1455 immunofluorescent images are representative of at least 3 independent

1456 repeats. Data in c represent mean \pm SEM. Each dot is an individual mouse.

a

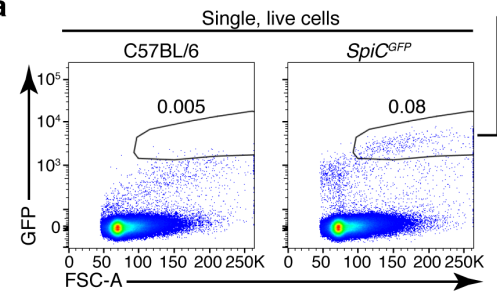

C

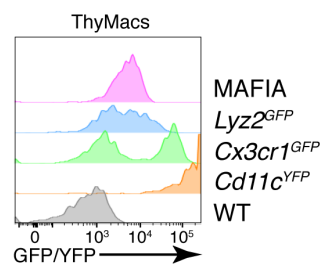

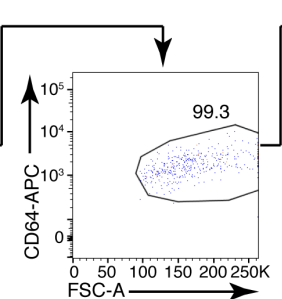

d

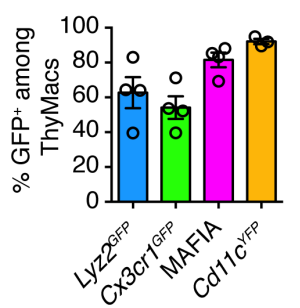

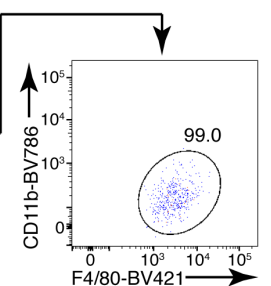

e

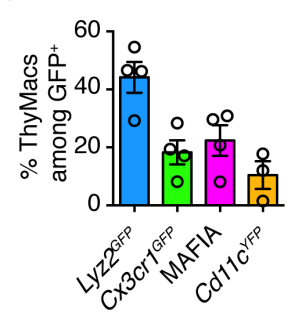

f

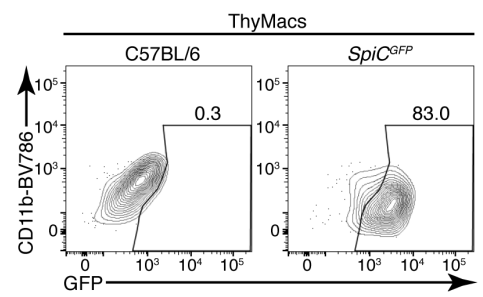

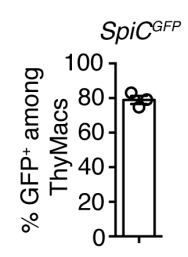

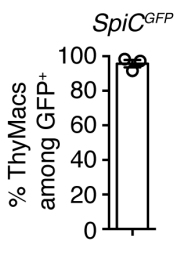


Figure 2 - Figure Supplement 1: Expression of Spic ${ }^{G F P}$ marks most

1458 thymic macrophages. a Example of the gating strategy to identify ThyMacs

1459 among Spic ${ }^{G F P+}$ cells. b Frequencies of ThyMacs among Spic ${ }^{G F P+}$ cells. c

1460 Representative flow cytometry plots of the expression of four reporter alleles

1461 in ThyMacs. d Frequencies of GFP/YFP+ cells among ThyMacs. e

1462 Frequencies of ThyMacs among GFP/YFP ${ }^{+}$cells. $\mathbf{f}$ Representative flow

1463 cytometry plots of the expression of Spic ${ }^{G F P}$ in ThyMacs. $g$ Frequencies of

1464 Spic $^{G F P+}$ cells among ThyMacs. All flow cytometry plots are representative of

1465 at least 3 independent repeats. Data in $\mathbf{b}, \mathbf{d}, \mathbf{e}$, and $\mathbf{f}$ represent mean $\pm S E M$.

1466 Each dot is an individual mouse. The numbers in the flow cytometry plots are

1467 the percent of cells in the respective gate.

1468

\begin{tabular}{lrr}
\multicolumn{3}{l}{$\begin{array}{l}\text { Table S1: Expression of differentially up-regular } \\
\text { thymic macrophages }\end{array}$} \\
\hline Gene name & ThyMacs & non-ThyMacs \\
\hline Irf7 & 3879.32 & 300.82 \\
Irf8 & 3528.27 & 1474.35 \\
Stat1 & 2403.69 & 522.04 \\
Dnmt3a & 1515.94 & 647.81 \\
Znxf1 & 1379.89 & 635.36 \\
Stat2 & 1210.35 & 472.53 \\
Nr1h3 & 1182.17 & 147.05 \\
Srebf1 & 975.09 & 399.06 \\
Rxra & 760.26 & 298.55 \\
Trps1 & 746.36 & 232.48 \\
Runx3 & 723.14 & 9.76 \\
Relb & 715.53 & 293.92 \\
Sp100 & 696.94 & 324.47 \\
Zbp1 & 639.19 & 69.83 \\
Tfec & 588.72 & 74.66 \\
Spic & 573.11 & 34.36 \\
Nfkbie & 569.74 & 226.76 \\
Ncoa4 & 550.69 & 249.15 \\
Rest & 548.22 & 269.22 \\
Meis3 & 530.8 & 120.91 \\
Bhlhe40 & 490.59 & 99.56 \\
Parp12 & 414.11 & 126.82 \\
Arid5b & 374.03 & 177.08 \\
Creb5 & 295.14 & 47.91 \\
Pparg & 276.54 & 33.24
\end{tabular}

1469 Figure 2 - Table Supplement 1: Expression of differentially up-

1470 regulated transcription factors in thymic macrophages. Transcription

1471 factors that were highly expressed in thymic macrophages (>250) and up- 
1472 regulated $>2$-fold in thymic macrophages compared to non-thymic

1473 macrophages were listed alphabetically, and the geometric mean of 4

1474 replicates of thymic macrophages (ThyMacs) and two replicates of each of the

14759 non-thymic macrophage populations (non-ThyMacs) were recorded. Non-

1476 thymic macrophages are: spleen red pulp macrophages, Kupffer cells,

1477 bronchoalveolar lavage macrophages, peritoneal cavity macrophages, aorta

1478 macrophages, heart macrophages, white adipose tissue macrophages,

1479 central nervous system microglia, spinal cord macrophages.

Table S2. List of the differentially expressed genes among Timd4+ thymic macrophages, Cx3cr1+ thymic macrophages, and thymic monocytes

\begin{tabular}{|c|c|c|c|c|c|}
\hline \multicolumn{2}{|c|}{ CX3CR1+ ThyMacs } & \multicolumn{2}{|c|}{ Tim4+ ThyMacs } & \multicolumn{2}{|c|}{ ThyMonos } \\
\hline Gene name & adjusted $p$-value & Gene name & adjusted $p$-value & Gene name & adjusted $p$-value \\
\hline Ctsz & 0 & Hpgd & 0 & Alox5ap & 0 \\
\hline Cd63 & 0 & Serpinb6a & 0 & s100a6 & 0 \\
\hline Pmepa1 & 0 & Slc40a1 & 0 & Ly6c2 & 0 \\
\hline Zmynd15 & 0 & Cd81 & 0 & Ifi27/2a & 0 \\
\hline Olfm/3 & 0 & Vcam1 & 0 & Fau & 0 \\
\hline Mmp2 & 0 & Cfp & 0 & Coro1a & 0 \\
\hline AU020206 & $1.60 \mathrm{E}-290$ & Spic & 0 & Ccr2 & 0 \\
\hline Plxnd1 & $1.59 \mathrm{E}-285$ & $T r f$ & 0 & Rps27 & 0 \\
\hline Cst7 & 8.68E-279 & Actn1 & 0 & Tmsb10 & 0 \\
\hline Dnase1/3 & $2.45 \mathrm{E}-270$ & Maf & 0 & Ifitm2 & 7.21E-302 \\
\hline Timp2 & $2.15 \mathrm{E}-267$ & Pld3 & 0 & Fxyd5 & 6.36E-299 \\
\hline Lgals3bp & 8.69E-263 & $/ / 18$ & 0 & Rps19 & 2.04E-292 \\
\hline Pdgfa & $6.87 \mathrm{E}-255$ & Mrc1 & 0 & $R p / 18$ & $6.50 \mathrm{E}-291$ \\
\hline Mmp14 & 2.33E-253 & Crip2 & 0 & $R p / 9$ & $1.11 \mathrm{E}-289$ \\
\hline Fam46c & $9.99 \mathrm{E}-235$ & Tmem65 & 0 & Rps23 & $1.28 \mathrm{E}-289$ \\
\hline Chst2 & 1.19E-226 & Igf1 & 0 & Napsa & 8.91E-279 \\
\hline$C p$ & 5.36E-225 & $E p b 41 / 3$ & 0 & $M s 4 a 4 c$ & 8.25E-272 \\
\hline Camk1 & $7.12 \mathrm{E}-225$ & Timd4 & 0 & Plac8 & 2.10E-270 \\
\hline$B 2 m$ & $1.09 \mathrm{E}-222$ & Blvrb & 0 & Rp/18a & $9.26 \mathrm{E}-269$ \\
\hline Lhfp/2 & $4.52 \mathrm{E}-217$ & Clec1b & 0 & S100a4 & 4.98E-268 \\
\hline Acp5 & $5.90 \mathrm{E}-216$ & Cd68 & 0 & Cd52 & 3.67E-267 \\
\hline Lag3 & $3.91 \mathrm{E}-213$ & $A x I$ & 0 & Rps14 & 1.94E-266 \\
\hline Lyz2 & $1.28 \mathrm{E}-209$ & Paqr9 & 3.32E-307 & Ifitm3 & 3.19E-263 \\
\hline H2-M2 & $1.22 \mathrm{E}-199$ & Sdc3 & $3.45 \mathrm{E}-305$ & $R p / 34$ & 2.02E-261 \\
\hline Psap & 7.26E-198 & Myo9a & 5.59E-305 & Rps27a & $3.67 \mathrm{E}-260$ \\
\hline Gatm & 1.33E-192 & Scp2 & 3.79E-302 & Rp/36 & $1.54 \mathrm{E}-259$ \\
\hline Cpd & $1.50 \mathrm{E}-192$ & Selenop & 2.10E-295 & Rps16 & $2.55 \mathrm{E}-258$ \\
\hline
\end{tabular}




\begin{tabular}{|c|c|c|c|c|c|}
\hline C3 & 2.34E-187 & Lrp1 & 2.08E-294 & Rp/24 & 1.37E-257 \\
\hline Cxcl16 & $8.11 \mathrm{E}-183$ & Lap3 & $1.45 \mathrm{E}-290$ & Rps9 & $6.34 \mathrm{E}-253$ \\
\hline Lgals3 & $1.57 \mathrm{E}-182$ & Marcks & 2.77E-279 & Gpr141 & $1.21 \mathrm{E}-246$ \\
\hline Ube2j1 & $1.63 E-180$ & Glul & 3.64E-279 & Rp/27a & 3.06E-243 \\
\hline Plxnc1 & $9.84 \mathrm{E}-180$ & Hebp1 & 3.76E-278 & $R p / 17$ & 8.15E-241 \\
\hline Stab1 & 4.07E-176 & Ear2 & 4.53E-276 & Rps24 & $1.46 \mathrm{E}-240$ \\
\hline Cyth1 & $3.27 \mathrm{E}-163$ & Apoc1 & $2.49 \mathrm{E}-275$ & Rps13 & $2.34 \mathrm{E}-236$ \\
\hline Spsb1 & $3.96 \mathrm{E}-163$ & Kcna2 & $3.72 \mathrm{E}-275$ & Rp/38 & 1.95E-226 \\
\hline Blnk & $2.35 \mathrm{E}-162$ & Myo10 & 9.05E-269 & $H 2-D M b 1$ & $1.02 \mathrm{E}-223$ \\
\hline$C \times 3 c r 1$ & $9.29 \mathrm{E}-162$ & Atp13a2 & $2.95 \mathrm{E}-267$ & Rps18 & 5.39E-223 \\
\hline Med10 & $5.25 \mathrm{E}-161$ & Slc1a3 & $6.24 \mathrm{E}-263$ & Rp/19 & 3.68E-221 \\
\hline Nek6 & $5.28 \mathrm{E}-160$ & $S / c o 2 b 1$ & $1.11 \mathrm{E}-258$ & Rp/8 & $2.01 \mathrm{E}-219$ \\
\hline Ptms & 1.05E-159 & $m t-N d 2$ & $3.45 \mathrm{E}-258$ & Rp/7a & 4.17E-217 \\
\hline Anxa5 & 1.10E-156 & Wwp1 & 2.16E-253 & Gm34084 & 5.23E-216 \\
\hline Gpnmb & $1.21 \mathrm{E}-154$ & Aplp2 & $4.22 \mathrm{E}-248$ & $R p / 13$ & $2.08 E-215$ \\
\hline $\operatorname{ltg} 55$ & $2.78 \mathrm{E}-154$ & Atp8a1 & 5.03E-248 & $R p / 11$ & $2.47 \mathrm{E}-213$ \\
\hline Муо5а & $1.11 \mathrm{E}-146$ & $P 2 r y 13$ & 3.17E-247 & Rp/35a & $2.13 E-210$ \\
\hline Runx3 & $1.81 \mathrm{E}-146$ & Ccdc148 & $4.70 \mathrm{E}-245$ & Rpsa & 1.62E-209 \\
\hline Tmem176a & $2.34 \mathrm{E}-144$ & Grn & $1.58 \mathrm{E}-244$ & Rp/6 & $5.70 \mathrm{E}-208$ \\
\hline Ctss & $4.81 \mathrm{E}-141$ & Bank1 & $1.82 \mathrm{E}-239$ & Tpt1 & 2.63E-206 \\
\hline $\operatorname{sh} 3 p x d 2 b$ & $9.38 \mathrm{E}-141$ & Mertk & $2.15 \mathrm{E}-238$ & Rack1 & $2.14 \mathrm{E}-203$ \\
\hline Rtcb & $4.42 \mathrm{E}-140$ & Nr1h3 & $1.13 E-235$ & Rp/23 & $6.14 \mathrm{E}-199$ \\
\hline Fam20c & $1.91 \mathrm{E}-139$ & Prnp & $2.93 \mathrm{E}-235$ & Rp/26 & 7.48E-198 \\
\hline II2rg & $8.84 \mathrm{E}-138$ & Ninj1 & $2.42 \mathrm{E}-234$ & Rps6 & $6.64 \mathrm{E}-197$ \\
\hline Lpcat2 & 8.53E-137 & Fcna & 3.33E-233 & Rps10 & 2.06E-195 \\
\hline Kynu & $8.49 \mathrm{E}-136$ & Csrp1 & $1.16 \mathrm{E}-230$ & ler5 & 1.06E-191 \\
\hline Tnfsf13b & 8.77E-136 & $R g / 1$ & 7.18E-229 & Rps3 & $8.23 E-185$ \\
\hline Gpr157 & $1.18 \mathrm{E}-135$ & $L p l$ & $4.94 \mathrm{E}-223$ & $R p / 27$ & $8.23 E-185$ \\
\hline Tgfbr1 & 7.63E-135 & Fam $213 b$ & $1.08 \mathrm{E}-222$ & Rps5 & 8.36E-185 \\
\hline$H 2-K 1$ & $1.15 \mathrm{E}-133$ & $T c f 7 / 2$ & $1.26 \mathrm{E}-222$ & Rps7 & $3.96 \mathrm{E}-182$ \\
\hline Basp1 & $1.23 \mathrm{E}-133$ & $A B 124611$ & 4.64E-221 & Rps15a & $6.82 \mathrm{E}-182$ \\
\hline Pla2g7 & $1.80 \mathrm{E}-132$ & $A b c c 3$ & $3.28 \mathrm{E}-216$ & Rps11 & $1.97 \mathrm{E}-180$ \\
\hline Fth1 & $4.19 \mathrm{E}-131$ & Fcgrt & $5.79 \mathrm{E}-216$ & $R p s 4 x$ & $5.07 E-180$ \\
\hline Ggh & $1.85 \mathrm{E}-126$ & Tgm2 & $1.88 \mathrm{E}-215$ & Rplpo & $3.09 \mathrm{E}-177$ \\
\hline Adam19 & $6.94 \mathrm{E}-126$ & Itgad & 5.35E-214 & Ly6i & 8.17E-176 \\
\hline C3ar1 & 7.35E-125 & Ptgs1 & $2.94 \mathrm{E}-213$ & S100a11 & $6.23 \mathrm{E}-175$ \\
\hline Ccl12 & 3.37E-123 & Laptm4a & $1.01 \mathrm{E}-212$ & Atox 1 & $1.22 \mathrm{E}-174$ \\
\hline Hven1 & $2.51 \mathrm{E}-121$ & Comt & 1.33E-206 & Pim1 & $9.56 \mathrm{E}-174$ \\
\hline Anxa3 & $8.60 \mathrm{E}-121$ & Creg1 & $3.24 \mathrm{E}-205$ & Sh3bgrl3 & $3.97 \mathrm{E}-173$ \\
\hline Tgfbi & $1.88 \mathrm{E}-120$ & Adgre1 & 9.67E-205 & Ciita & 7.35E-173 \\
\hline Ctsd & $2.73 \mathrm{E}-117$ & Clec12a & $6.33 E-204$ & Eef1a1 & $6.09 \mathrm{E}-172$ \\
\hline $\operatorname{ltm} 2 c$ & $5.19 \mathrm{E}-116$ & Tspan4 & 7.80E-203 & Rps3a1 & $9.09 E-168$ \\
\hline Tmem119 & $5.62 \mathrm{E}-116$ & Txn1 & $9.13 E-203$ & Gm2a & $6.07 \mathrm{E}-165$ \\
\hline Rap2a & $1.03 E-114$ & $C t s b$ & $9.52 \mathrm{E}-201$ & Ptprc & $2.05 \mathrm{E}-163$ \\
\hline Ctsl & $4.00 \mathrm{E}-114$ & Mrap & 5.65E-197 & Rp/37 & $1.51 \mathrm{E}-161$ \\
\hline $\operatorname{ltg} a 6$ & $1.83 \mathrm{E}-113$ & S/c16a9 & $5.99 \mathrm{E}-197$ & Rps25 & $3.03 E-160$ \\
\hline
\end{tabular}




$\begin{array}{lrl}\text { B4galnt1 } & 2.45 \mathrm{E}-113 & \text { Abcg3 } \\ \text { Fam3c } & 1.64 \mathrm{E}-112 & \text { Pla2g15 } \\ \text { Tmem173 } & 1.54 \mathrm{E}-111 & \text { C1qc } \\ \text { Ski } & 3.59 \mathrm{E}-111 & \text { Agpat3 } \\ \text { Anpep } & 5.85 \mathrm{E}-111 & \text { Hs6st1 } \\ \text { Gng2 } & 2.37 \mathrm{E}-110 & \text { Dmpk } \\ \text { Nceh1 } & 2.88 \mathrm{E}-110 & \text { Cd38 } \\ \text { H2-Q7 } & 4.94 \mathrm{E}-108 & \text { Tmem26 } \\ \text { Rtn1 } & 1.28 \mathrm{E}-106 & \text { Slc11a1 } \\ \text { Sorl1 } & 1.31 \mathrm{E}-103 & \text { Cd300a } \\ \text { Glipr1 } & 1.22 \mathrm{E}-102 & \text { Slc7a7 } \\ \text { Gsn } & 2.00 \mathrm{E}-102 & \text { Cyb5a } \\ \text { Afdn } & 4.54 \mathrm{E}-102 & \text { Sipa1/1 } \\ \text { Ak2 } & 1.11 \mathrm{E}-101 & \text { Il18bp } \\ \text { Ntpcr } & 2.21 \mathrm{E}-98 & \text { Cd86 } \\ \text { Scarb2 } & 3.16 \mathrm{E}-97 & \text { Vamp5 } \\ \text { Creb5 } & 5.41 \mathrm{E}-97 & \text { Jup } \\ \text { Gsto1 } & 5.56 \mathrm{E}-97 & \text { Blvra } \\ \text { Ncf1 } & 4.26 \mathrm{E}-96 & \text { Mgst1 } \\ \text { Ppfia4 } & 4.97 \mathrm{E}-96 & \text { Tbxas1 } \\ \text { Chchd10 } & 7.77 \mathrm{E}-96 & \text { Hpgds } \\ \text { Gna12 } & 1.23 \mathrm{E}-95 & \text { Tgfbr2 } \\ \text { Mvb12b } & 1.80 \mathrm{E}-95 & \text { Clec4n } \\ \text { Rasal3 } & 1.45 \mathrm{E}-94 & \text { Ms4a7 } \\ \text { Scoc } & 6.86 \mathrm{E}-94 & \text { Sirpa } \\ \text { Cfb } & 6.00 \mathrm{E}-93 & \text { Fyn } \\ \text { Lmna } & 1.04 \mathrm{E}-92 & \text { Cadm1 }\end{array}$

\begin{tabular}{|c|c|}
\hline & H3fза \\
\hline & Btg2 \\
\hline 92 & Rp/15 \\
\hline 91 & Cnn2 \\
\hline $95 \mathrm{E}-1$ & Cdkn1 \\
\hline 91 & Slfn1 \\
\hline $79 \mathrm{E}-1$ & Sem1 \\
\hline 02E-189 & Lsp1 \\
\hline 05E-188 & $R p / 37 a$ \\
\hline 41E-187 & Rp/22 \\
\hline $28 \mathrm{E}-1$ & Sirpb1 \\
\hline 94E-187 & Traf1 \\
\hline 87 & $E m b$ \\
\hline 48E-186 & Rp/30 \\
\hline $52 E-183$ & Rps15 \\
\hline 05E-183 & $H 2-A b$ \\
\hline 69E-182 & $\| 11 b$ \\
\hline 30E-178 & Rps28 \\
\hline 48E-178 & Jarid2 \\
\hline $.47 \mathrm{E}-177$ & Rps26 \\
\hline 04E-177 & Rp/32 \\
\hline $2.70 \mathrm{E}-176$ & Pld4 \\
\hline $3.52 E-175$ & Cbfa2 \\
\hline $5.30 \mathrm{E}-175$ & Rps21 \\
\hline 3.35E-171 & $F g r$ \\
\hline $284 \mathrm{~F}-168$ & Rps \\
\hline & \\
\hline
\end{tabular}

5.92E-159

$1.14 \mathrm{E}-158$

$1.42 \mathrm{E}-158$

1.09E-156

2.57E-156

4.83E-155

4.08E-154

1.34E-152

$1.78 \mathrm{E}-152$

3.64E-152

4.81E-152

6.97E-152

4.22E-151

1.32E-147

1.14E-146

2.84E-145

3.05E-145

4.52E-145

1.82E-143

1.53E-142

4.21E-142

9.07E-142

$1.54 \mathrm{E}-141$

4.04E-141

4.04E-141

1.11E-139

5.34E-138

Figure 5 - Table Supplement 1: List of the differentially expressed

1482 genes among $\mathrm{Timd}^{+}$thymic macrophages, $\mathrm{C} \times 3 \mathrm{cr} \mathbf{1}^{+}$thymic

1483 macrophages, and thymic monocytes. The top 100 differentially expressed

1484 genes among the three clusters are listed by their negative $\log _{10}$ transformed 1485 p-value. 